\title{
Particle jets in colliding two ultraintense laser pulses of varying frequencies
}

\author{
Siddharth Tiwary ${ }^{*}{ }^{*}$ \\ Physics Department, Indian Institute of Technology Bombay, Mumbai, Maharashtra 400076, India \\ Naveen Kumar (1) $^{\dagger}$ \\ Max-Planck-Institut für Kernphysik, Saupfercheckweg 1, D-69117 Heidelberg, Germany
}

(Received 9 April 2021; accepted 23 November 2021; published 17 December 2021)

\begin{abstract}
We present results on the evolution of jets (electrons-positrons and photons) formed in colliding two ultraintense laser pulses with varying frequencies and amplitudes. We focus on the nonlinear dynamics of the seeded electrons in the superposition of the two counterpropagating laser pulses and its implications for the experimentally measurable properties of jets, e.g., angular spectra and widths of the emitted jets, by changing the intensity and frequency of the two colliding laser pulses. We verify our analytical results by doing both Monte Carlo and two-dimensional particle-in-cell simulations.
\end{abstract}

DOI: 10.1103/PhysRevResearch.3.043190

\section{INTRODUCTION}

Generating and studying the dynamics of electron-positron $(e-p)$ plasma in a laboratory setup are important not only for understanding several astrophysical objects such as pulsars, relativistic jets, and gamma-ray bursts (GRBs) where $e-p$ plasmas are expected to be present, but also for verifying the most important predictions made by quantum electrodynamics (QED) on the breakdown of a vacuum leading to the generation of $e-p$ plasmas [1-12]. With the commissioning of 10-PW laser systems around the world, the experimental feasibility of studying the $e-p$ plasmas in a laboratory is an exciting possibility [13-16]. Though the generation of $e-p$ pairs has been achieved in a laboratory [17-19], however, the generation of $e-p$ plasmas directly by laser pulses can enable studying properties of these plasmas in an all-optical setup [20,21]. This can pave the way to design laboratory astrophysics experiments studying the physical processes occurring in diverse astrophysical scenarios, such as the generation of polarized light in GRBs [22].

For testing the validity and predictions of the QED theory in a nonlinear regime, the setups of laser-laser collisions (to initiate an electromagnetic cascade from a seed electron) [5,6,20,23], beam-beam collisions [24], and laser-beam collisions [25] have been studied extensively in the last few years. The first can mimic both shower- and avalanche-type cascades [12]. Of particular interest are the spectra of particles generated in this setup that can be experimentally detected to verify

\footnotetext{
*siddharth110200@gmail.com

†Corresponding author: naveen.kumar@mpi-hd.mpg.de
}

Published by the American Physical Society under the terms of the Creative Commons Attribution 4.0 International license. Further distribution of this work must maintain attribution to the author(s) and the published article's title, journal citation, and DOI. Open access publication funded by the Max Planck Society. $e-p$ generation via the Breit-Wheeler process in an intense laser field [17,18,26,27]. Incidentally, the classical motion of an electron in this setup exhibits features of nonlinear dynamics [28-32]. Indeed, the motion of the electron in a standing wave formed by two laser pulses is highly nonlinear, though it exhibits some attractor-type solutions that can be exploited to study the cascade formation in a laboratory setup. There have also been attempts to study the generation of pairs in a two-color laser pulse setup [33-35]. Also, combining an optical laser pulse with an X-ray laser pulse can facilitate copious amounts of pair production in a laboratory since the high-photon energy associated with an $\mathrm{x}$ ray can help dynamically lower the Schwinger barrier [7,8,33,35-37].

As mentioned before, the electromagnetic cascade initiated by two optical laser pulses has been studied extensively in various setups. However, the setup of two counterpropagating laser pulses with different frequency ratios has attracted rather brief attention [28]. Not only do the ultrahigh-intensity laser pulses have broadband spectra, but also the laser pulse frequency can change due to nonlinear plasma effects $[25,38$ 40]. Thus this setup can be a natural setting for studying the cascade in counterpropagating optical laser pulses. It can also be relevant for studying the cascade in a setup where an optical laser pulse is paired with an X-ray laser pulse. [41,42]. Moreover, this setup may also serve as a valuable tool to control the spectra of the particles generated in an electromagnetic cascade. The spectra of particles generated in a vacuum breakdown by two noncollinear laser pulses has been studied [7,8]. However, the directionality and collimation of the cascade particles from a vacuum were explained using kinematic arguments on the basis of the Lorentz transformations involved in moving from the rest frame of the particles to the laboratory frame of reference.

In this paper, we study the electromagnetic cascade in two counterpropagating laser pulses of varying frequency and amplitude ratios. In particular, we focus on the properties of jets formed in the electromagnetic cascade initiated by an electron 
or positron in the focus of these colliding pulses. Thus our setup and goals are different from Refs. [7,8], and we explain the directionality and collimation of the particle beams based on the nonlinear dynamics of the electron in the focus of the two laser pulses. In particular, we propose that our setup can be used to control the directionality of the particle and photon beams in a laboratory experiment. The remainder of this paper is organized as follows: In Sec. II, we discuss the setup, followed by an analysis of the nonlinear dynamics of an electron in the standing wave created by two counterpropagating laser pulses of varying frequencies in Sec. III, where we derive the angle of emission of particles in the classical limit. In Sec. IV, we show results on jet formation and angular spectra from a Monte Carlo code evolving all three components of positions and momenta. Afterwards, we present the results of two-dimensional (2D) simulations from a particle-in-cell (PIC) code, SMILEI, in Sec. V.

\section{PHYSICAL SETUP}

Figure 1 shows the physical setup in our case. For our theoretical analysis, we employ a cgs system with natural units, i.e., $m_{e}=e=c=k_{B}=1$, where $c$ is the velocity of the light in a vacuum, $e$ is the elementary charge, $k_{B}$ is the Boltzmann's constant, and $m_{e}$ is the electron mass. When stating numerical values for frequency and wavelength, we normalize time and length to a relevant reference frequency $\omega_{L}$ or the corresponding wavelength $\lambda_{L}$. These values are $\lambda_{L}=1 \mu \mathrm{m}$ and $\omega_{L}=2 \pi c / \lambda_{L}$, corresponding to the existing and upcoming state-of-the-art laser systems [13-16]. We use laser pulses lasting a few tens of laser cycles, in accordance with the existing and upcoming laser facilities. For comparison, the Vulcan facility is expected to deliver short pulses of $1 \mathrm{PW}$ power at $1.053 \mu \mathrm{m}$ and $\tau_{L}=500 \mathrm{fs}$ [15] (140 wave periods). The 10-PW facility, L4, of the Extreme Light Infrastructure (ELI) project is supposed to deliver pulses with $\tau_{L}=140 \mathrm{fs}$ duration (40 laser cycles), while the 1-PW L3 beamline promises short pulses $\left(\tau_{L}<30 \mathrm{fs}\right.$, ten laser cycles) at $800 \mathrm{~nm}$ wavelength [14].

We use two counterpropagating electromagnetic waves, either linearly (LP) or circularly polarized (CP) with opposite helicity, propagating along the $x$ axis, with pulse durations $\tau$. For the purpose of investigating the nonlinear dynamics and for Monte Carlo simulations, these waves are assumed to have uniform spatiotemporal profiles. This is valid as long as $r_{0} \gg \lambda_{L}$, where $r_{0}$ is the spot size of the laser pulse. However, this assumption is relaxed in PIC simulations (see Sec. V). The motion of charged particles (with charge $\pm e$ ) in an electromagnetic field $A_{\mu}$ is frequently characterized by several dimensionless parameters [29]: The quantity $a=\sqrt{A_{\mu} A^{\mu}}$ [26] is Lorentz invariant and represents the electron quiver momentum normalized to $m_{e} c$. When $a=1$, the work done by the laser field in a distance $\lambda / 2 \pi$ equals $m_{e} c^{2}$. Thus $a=1$ represents the threshold for the relativistic regime of the laserplasma interaction. For $a=\epsilon_{\mathrm{rad}}^{-1 / 3}$, one has to take into account the effect of the radiation reaction force on the electron dynamics since the recoil caused by the emitted photon on the electron becomes significant, affecting the electron dynamics in a laser field. The parameter $\epsilon_{\mathrm{rad}}=2 \omega / 3$ (in normalized units, $\omega$ is the laser frequency) is proportional to the ratio

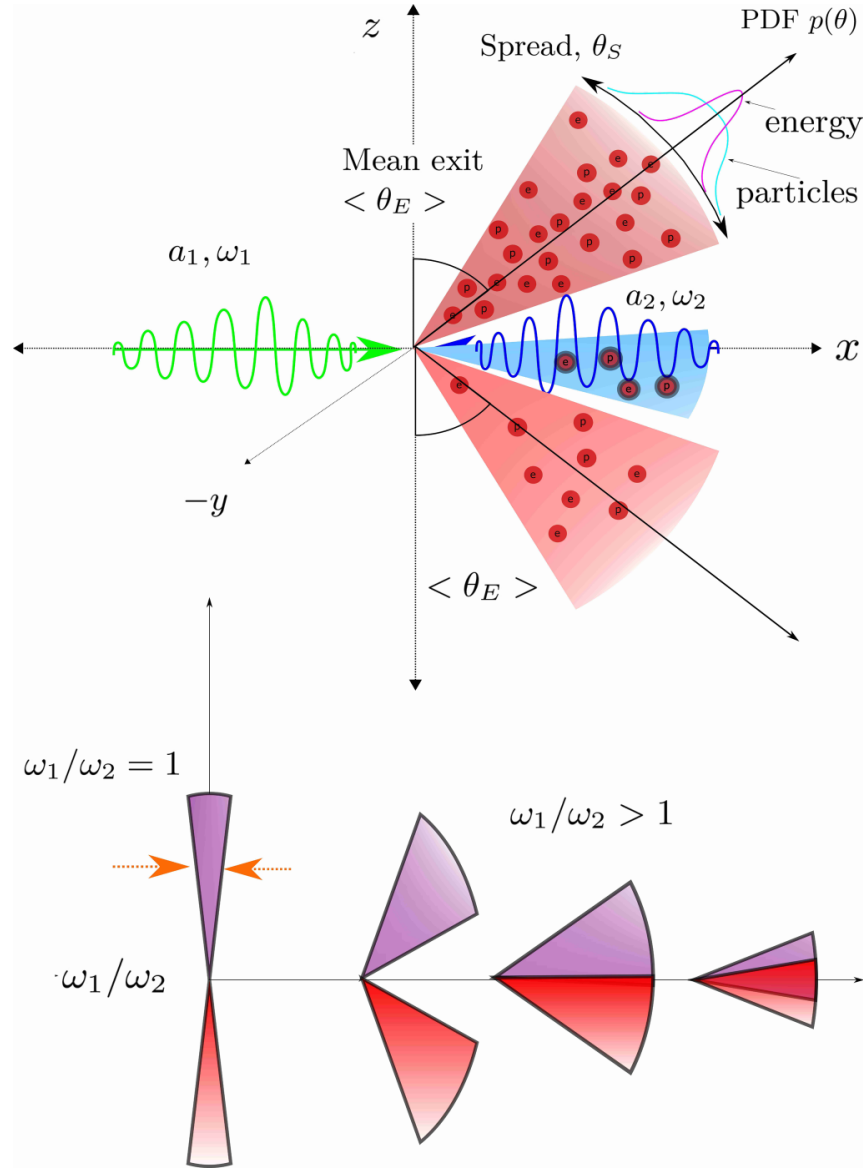

FIG. 1. Top: A schematic of colliding counterpropagating linearly polarized (LP) laser pulses showing the particle distribution function (PDF) of emitted electrons and positrons. The energy distribution (pink curve) is narrower than the corresponding PDF of particles (cyan curve) in the cascade jet (red), which is pancaked in the $x$ - $z$ plane for motion in planar waves. The mean emission angle $\theta_{E}$ is measured from the transverse plane, and the spread of the jet, $\theta_{S}$, is calculated as the standard deviation of the probability distribution. The vacuum-decay jet (blue), observed for fields approaching the Schwinger critical value, lies in the $x-y$ plane, perpendicular to the electric field. As the particles in the jet produced by vacuum decay begin interacting with the laser field, they accelerate and merge with the cascade jets. Bottom: The variation in the jet, as the frequency ratio is varied while maintaining a constant amplitude ratio. Orange arrows indicate what we postulate is the effect of phase space contraction (order), which is prominent for the equal-frequencies case. The upper and lower jets gradually merge.

of the classical electron radius to the laser wavelength [29]. The gauge-invariant parameter describing the multiphoton Compton effect, $\chi_{e}=\hbar\left|F^{\mu v} p_{v}\right|$, for a particle with velocity $\mathbf{v}$, four-momentum $p_{v}$, and Lorentz factor $\gamma$ in electric and magnetic fields $\mathbf{E}$ and $\mathbf{B}$, respectively, with the electromagnetic tensor $F^{\mu \nu}=\partial^{\mu} A^{\nu}-\partial^{\nu} A^{\mu}$, is one other such parameter. Similarly, one can define a gauge-invariant parameter for photons, $\chi_{\gamma}=\hbar^{2}\left|F^{\mu v} k_{v}\right|$, with $\hbar k_{v}=\left(\hbar \omega_{\gamma}, \hbar \mathbf{k}_{\gamma}\right)$ being the four-momentum of the photon undergoing the Breit-Wheeler process. QED effects become important for $\chi_{e} \simeq 1$ [26].

We employ the Lorenz gauge, $\mathbf{B}=\nabla \times A$ and $\mathbf{E}=$ $-\partial \mathbf{A} / \partial \mathbf{t}$. We label the frequency of the wave traveling towards 
the positive $x$ direction $\omega_{1}$, and that of the oppositely traveling wave is labeled $\omega_{2}$. The corresponding amplitudes of the two waves are labeled $a_{1}$ and $a_{2}$, respectively, with the amplitude ratio defined as $\lambda \equiv a_{2} / a_{1}$.

We use both linearly and circularly polarized counterpropagating laser pulses. For the case of linear polarization, the vector potential reads $\mathbf{A}=\left\{a_{1} \cos [\omega(x-t)]+a_{2} \cos [\omega(x+\right.$ $t)]\} \hat{\mathbf{z}}=a_{0}\{\cos [\omega(x-t)]+\lambda \cos [\omega(x+t)]\} \hat{\mathbf{z}} / 2$. For circularly polarized waves, this will instead be $\mathbf{A}=$ $\left.a_{1}\{\cos [\omega(x-t)] \hat{\mathbf{y}}+\sin [\omega(x-t)] \hat{\mathbf{z}}]\right\}+a_{2}\{\cos [\omega(x+t)] \hat{\mathbf{y}}+$ $\sin [\omega(x+t)] \hat{\mathbf{z}}]\}=a_{0}(\{\cos [\omega(x-t)]+\lambda \cos [\omega(x+t)]\} \hat{\mathbf{y}}+$ $\{\sin [\omega(x-t)]+\lambda \sin [\omega(x+t)]\} \hat{\mathbf{z}}) / 2$. For our analysis in Secs. IV and $\mathrm{V}$, we have four degrees of freedom to manipulate: $a_{1}, a_{2}, \omega_{1}$, and $\omega_{2}$. We frequently fix either two of the amplitudes, or two of the frequencies (wavelengths), while constraining the other two quantities and holding their product or ratio constant. Here, we have defined $a_{0} \equiv 2 a_{1}$, so that $a_{2}=\lambda a_{1}$. For studying and analyzing results, we often invoke the zero-momentum frame (ZMF, also called the zero-motion frame), with velocity $\boldsymbol{v}_{\mathrm{ZMF}} \equiv\left(\omega_{1}-\omega_{2}\right) /\left(\omega_{1}+\omega_{2}\right) \hat{\mathbf{x}}$. In this frame, each of the counterpropagating waves has a frequency $\omega \equiv \sqrt{\omega_{1} \omega_{2}}$, resulting in a degree of symmetry. Please note that for $\omega_{1}=\omega_{2}, \boldsymbol{v}_{\mathrm{ZMF}}=0$. This scenario coincides with the general case of moving into a frame of reference with the velocity equal to the longitudinal drift velocity of an electron in a single intense laser field propagating in the $\hat{x}$ direction [43]. For a proper choice of initial conditions, one can obtain self-similar motion of the electron in a single laser pulse [44]. This can also be formally equivalent to the Akhiezer-Polovin solution for circularly polarized electromagnetic waves in plasmas [45].

To study the nonlinear dynamics of an electron or positron in the classical limit, we resort to the Landau-Lifshitz (LL) prescription of the radiation reaction [43]. The LL approximation is valid as long as $\chi_{e} \ll 1$. However, if we only refer to the classical motion of these pairs in the laser field, then one can still use the LL equation for the particle dynamics as long as $\chi_{e} \leqslant 1$ in the laser field. These particles generated in the laser field are to be accelerated for a short time before their $\chi_{e}$ parameter becomes large and the LL approximation fails. Nevertheless, one can still gain insight into the motion of these particles after their birth and until the time their $\chi_{e}$ becomes large. The parameters used in this section have been verified to ensure that $\chi_{e} \lesssim 10^{-3}$ during the simulation period, validating the use of the classical approximation. Denoting $\mathbf{u}=\left(u_{x}, u_{y}, u_{z}\right)=\gamma\left(v_{x}, v_{y}, v_{z}\right)$ as the four-velocities, the general equation of motion for a positron reads

$$
\begin{aligned}
\frac{d \mathbf{u}}{d t}= & (\mathbf{E}+\mathbf{v} \times \mathbf{B})-\frac{2}{3} \mathbf{v} \gamma^{2}\left[(\mathbf{E}+\mathbf{v} \times \mathbf{B})^{2}-(\mathbf{v} \cdot \mathbf{E})^{2}\right] \\
& +\frac{2}{3} \gamma\left(\left[\frac{\partial}{\partial t}+\mathbf{v} \cdot \nabla\right] \mathbf{E}+\left\{\mathbf{v} \times\left[\frac{\partial}{\partial t}+\mathbf{v} \cdot \nabla\right] \mathbf{B}\right\}\right) \\
& +\frac{2}{3}\{\mathbf{E} \times \mathbf{B}+[\mathbf{B} \times(\mathbf{B} \times \mathbf{v})]+\mathbf{E}(\mathbf{v} \cdot \mathbf{E})\} .
\end{aligned}
$$

As previously noted, we use the natural system of units, i.e., $m_{e}=e=c=1$. The first term in Eq. (1) is associated with the usual radiation pressure due to optical laser photons, while the remaining radiation reaction force terms account for the recoil caused by the emitted $\gamma$-ray photons on the electrons. We solve this equation and look at attractor-type solutions in different frames of reference. Another perspective or mechanism which we shall use to argue from is that of nonlinear dynamics, where we shall think of fermions getting swept up into and carried along attractors. In this context, we shall refer to the zero-motion frame (ZMF), where the frequencies are equal, the zero-power frame (ZPF), where the power flow or Poynting vector vanishes (reduces to ZMF for equal amplitudes), and the field rest frame (FRF), where the total (integrated) momentum and energy flow vanish. In a number of cases, this equation lends itself to approximate analytically tractable solutions. One- and two-dimensional motion in the electric antinodal plane of a standing wave leads, in particular, to illustrative solutions discussed in the Appendix. We study this equation for our setup next in Sec. III and show the features of nonlinear dynamics of a charged particle in the standing wave created by two counterpropagating laser pulses with unequal frequencies.

\section{NONLINEAR DYNAMICS AND EXIT ANGLE OF PARTICLES}

Nonlinear dynamics of an electron in the general case of two counterpropagating laser pulses has been studied in the past. The electron motion displays ordered, stochastic, and chaotic dynamics. The system exhibits a variety of attractor-type solutions, from limit cycles to strange attractors [28-30,46] characterizing chaotic motion. For $\lambda \neq 1$, Kirk analytically approximated the properties of limit cycles [28]. For this system, we consider attracting sets (attractors) in the phase space with particles performing bounded motion in their neighborhoods [47]. Note that from the very definition of an attracting set, there must exist a neighborhood in phase space such that all particles in this basin of attraction converge to the attracting set [48]. If we vary the frequencies of two electromagnetic waves $\omega_{1}$ and $\omega_{2}$ adiabatically, keeping their product constant, the attracting set is carried along with the $\mathrm{ZMF}$. Since $v_{\mathrm{ZMF}}=v_{\mathrm{ZMF}}\left(\omega_{1} / \omega_{2}\right)=\left(\omega_{1}-\omega_{2}\right) /\left(\omega_{1}+\omega_{2}\right)$ changes adiabatically, the particles always remain in the neighborhood of the (speeding or slowing) attractor and converge to it. Thus, if $\omega \equiv \sqrt{\omega_{1} \omega_{2}}$ is fixed, the attractor always looks the same in the ZMF, since the laser frequencies are fixed at $\omega$ in the ZMF. The attractor does not undergo reshaping, or disappear altogether. Particles trapped in the neighborhood of an attractor thus remain trapped, performing (bounded) motion in the same attractor. They do not jump to another attractor or another mode of motion, bounded or otherwise, since the frequencies are invariant in the ZMF. We refer to this proposed phenomenon as attractor dragging. For a limit cycle in the position space, we thus expect a circle in the ZMF to be a helix in the laboratory frame. Let us define the laboratory coordinates as $(t, x, y, z)$, and the coordinates in the ZMF as $\left(t^{\prime}, x^{\prime}, y, z\right)$. Keeping the parameters $a_{1}$ and $a_{2}$ unchanged (Lorentz invariant for plane waves) in the following, consider an attractor formed when the frequencies of both waves are $\omega$. Suppose the points in the attractor satisfy $f_{\omega, \omega}(t, x, y, z, d x / d t, d y / d t, d z / d t)=0$. Then, on using the frequencies $\omega_{1}$ and $\omega_{2}$, while keeping their product constant, the attractor follows $f_{\omega, \omega}\left(t^{\prime}, x^{\prime}, y, z, d x^{\prime} / d t^{\prime}, d y / d t^{\prime}, d z / d t^{\prime}\right)=0$ in the ZMF. 
This may be expressed in terms of the older septuplet $(t, x, y, z, d x / d t, d y / d t, d z / d t)$ in the laboratory frame, using $v_{\mathrm{ZMF}}$, the corresponding $\gamma_{\mathrm{ZMF}}$, and the Lorentz transforms $x^{\prime}=\gamma_{\mathrm{ZMF}}\left(x-v_{\mathrm{ZMF}} t\right)$ and $t^{\prime}=\gamma_{\mathrm{ZMF}}\left(t-v_{\mathrm{ZMF}} x\right)$. Thus the attractor in the laboratory frame $f_{\omega_{1}, \omega_{2}} \equiv$ $f_{\omega, \omega}\left(t^{\prime}, x^{\prime}, y, z, d x^{\prime} / d t^{\prime}, d y / d t^{\prime}, d z / d t^{\prime}\right)=0$.

Figures 2(a) and 2(b) show an attractor in the transverse $u_{y}-u_{z}$ plane and the attractor dragging, respectively, for circularly polarized waves having opposite helicity. This attractor is based on a limit cycle in the momentum space (electric nodal plane) but drifts in the position space. Figure 2(b) shows the trajectories of the same particle for a chosen set of initial conditions, as the frequencies of the two electromagnetic waves are varied. Figure 2(c) shows the evolution of the longitudinal velocity of the electron as the frequency ratio is varied with time, i.e., $\omega_{1}(t)=\omega\left(1+2 \times 10^{-3} \omega t\right)$ and $\omega_{2}(t)=\omega^{2} / \omega_{1}(t)$, where $\omega \approx 10.7 \omega_{L}$. When the frequency ratio $\omega_{2} / \omega_{1} \neq 1$, this implies a nonzero velocity of the ZMF, thus showing the dragging of the attractor formed for the ratio $\omega_{2} / \omega_{1}=1$. As expected for the frequency ratio becoming zero, one recovers the case of an electron motion in a single laser pulse showing an ultrarelativistic drift along the $\hat{x}$ direction. For $\omega_{2} / \omega_{1} \neq 1$, the attractor has a finite $v_{\mathrm{ZMF}}$ and moves along $x$. In other words, the limit cycle (and the attractor it is a part of (see Ref. [29]); in the position space, the limit cycle drifts, and it actually moves along the spiral arm in Fig. 2(b), which shows this scenario on a larger scale) moves along with the ZMF.

When the laser pulse ends, the (previously) trapped particle is traveling at a certain angle to the wave propagation axis. For the simplified scenario of plane waves under consideration, it is the exit angle (mean value) which we are interested in controlling. Specifically, when $\omega_{1}=\omega_{2}=\omega$, trapped particles remain trapped, statistically, in several different attracting sets. The mean longitudinal velocity is $v_{\mathrm{ZMF}}=0$ due to symmetry. As the frequencies are varied, the mean radial velocity after time $\tau$ is $v_{r}$. As $\omega_{1}$ is adiabatically increased while decreasing $\omega_{2}$ to keep $\omega=\sqrt{\omega_{1} \omega_{2}}$ constant, the ZMF speeds up to $\left(\omega_{1}-\omega_{2}\right) /\left(\omega_{1}+\omega_{2}\right)=\left(\omega_{1}^{2}-\omega^{2}\right) /\left(\omega_{1}^{2}+\right.$ $\left.\omega^{2}\right)$. Thus, in the laboratory frame, the final velocity is $\left(v_{\mathrm{ZMF}}, \sqrt{1-v_{\mathrm{ZMF}}^{2}} v_{r}\right)$. If $v_{r} \approx 1$, a central value for the final angle of emergence, as measured from the $y-z$ plane, reads as

$$
\theta_{E}=\tan ^{-1}\left(\frac{v_{\mathrm{ZMF}}}{\sqrt{1-v_{\mathrm{ZMF}}^{2}}}\right)=\tan ^{-1}\left(\frac{\omega_{1}-\omega_{2}}{2 \sqrt{\omega_{1} \omega_{2}}}\right) .
$$

This causes the exit angle to vary, as plotted in Fig. 2(d). As the frequencies are slowly changed and the ZMF speeds up, it drags along the limit cycle attractor, along with particles trapped inside. In a general scenario, with initial mean velocities $\left(v_{x}, v_{r}\right)$, the central final direction of emergence would be $\arctan \left[\left(v_{\mathrm{ZMF}}+v_{x}\right) /\left(\sqrt{1-v_{\mathrm{ZMF}}^{2}} v_{r}\right)\right]$. This may also be derived from a dynamical perspective, where $v_{\mathrm{ZMF}}$ turns out to be the requisite longitudinal velocity such that the total net momentum flow or Poynting vector from both the laser pulses vanishes. The second perspective will be important when we look into the effect of unequal amplitudes [49]. Analytically, we can also expect the angular energy spectrum to follow a

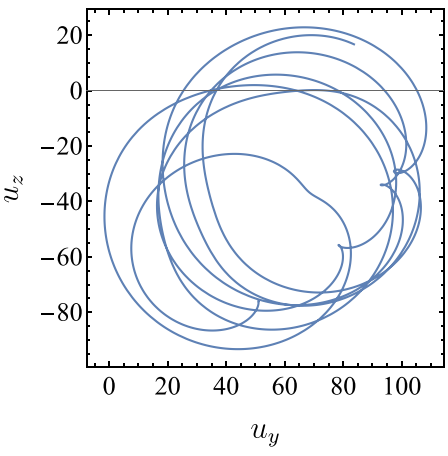

(a)

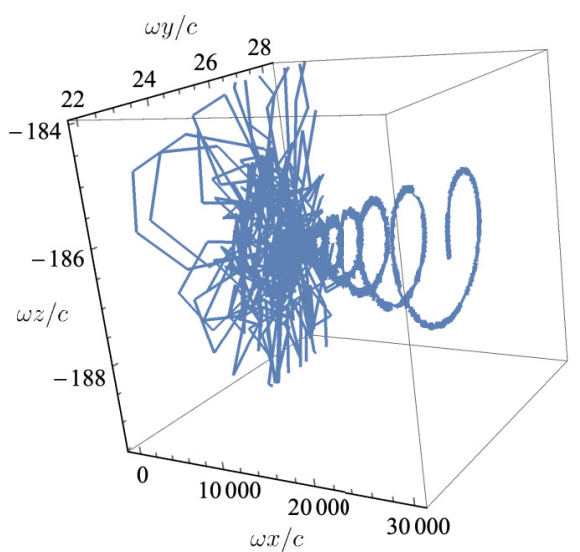

(b)

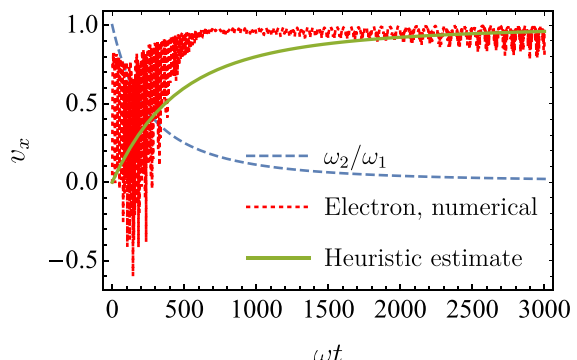

(c)

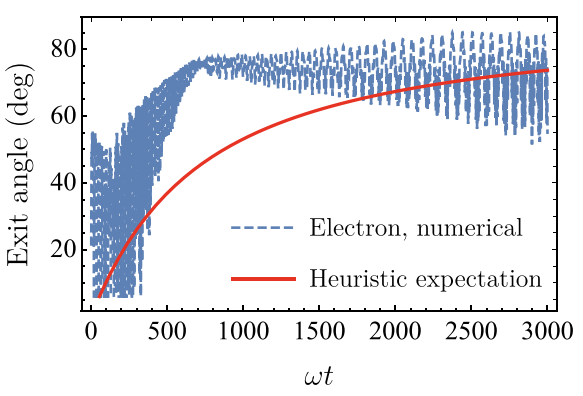

(d)

FIG. 2. Electron motion in $\mathrm{CP}$ laser pulses, with initial parameters $\lambda=0.2, \omega \approx 10.7 \omega_{L}, a_{0}=83.6, u_{x 0}=u_{z 0}=y_{0}=z_{0}=0$, $u_{y 0}=33.5$, and $x_{0}=4.97 /(2 \pi) \lambda_{L}$. (a) The limit cycle in the $u_{y}-u_{z}$ plane. (b) Trajectory with frequency variation $\omega_{1}(t)=\omega(1+2 \times$ $\left.10^{-3} \omega t\right)$ and $\omega_{2}(t)=\omega^{2} / \omega_{1}(t)$; details of the position space motion are not clearly visible, but the gradual movement of particles is. (c) $v_{\mathrm{ZMF}}$ and the longitudinal velocity, with $\omega_{2}(t) / \omega_{1}(t)$ overlaid. (d) Exit angle, computed from Eq. (2). 


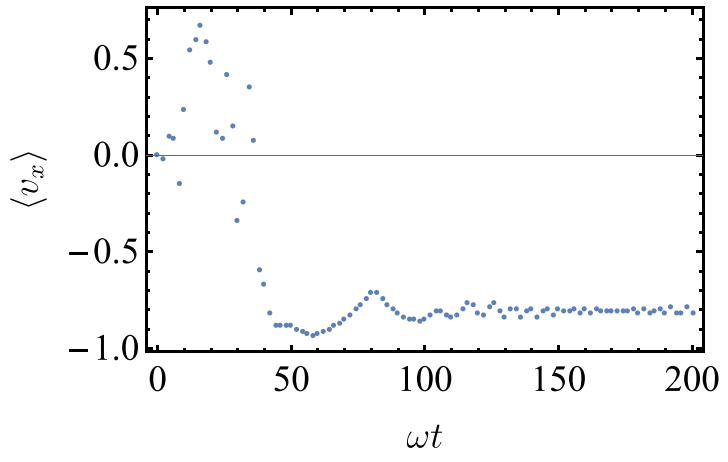

(a)

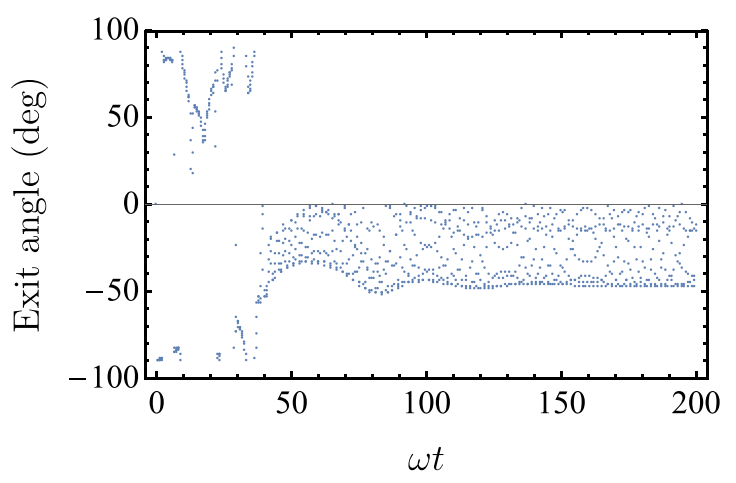

(b)

FIG. 3. Motion in LP laser pulses: $\lambda=1, \omega=7.10 \omega_{L}, a_{0}=$ 200, $u_{x 0}=u_{y 0}=u_{z 0}=y_{0}=z_{0}=0$, and $x_{0} \approx 0$. (a) Mean longitudinal velocity vs time, for $\omega_{1}(t)=\omega\left(1+5 \times 10^{-3} \omega t\right), \omega_{2}(t)=$ $\omega^{2} / \omega_{1}(t)$. (b) Exit angle vs time. Although the scattering is significant, a trend is clearly visible in the plot.

similar trend. As for the spread or angular width of the jet, it can be expected to be maximum for equal frequencies and decrease as the frequency ratio deviates from 1 . Equivalently, if the frequencies were equal but the resultant jet was seen from a moving reference frame, the jets would appear narrower.

In the case of linearly polarized waves, it is difficult to trap particles indefinitely. However, we consider the case of frequency variation and display the results in Fig. 3. The trajectory under examination (not shown) lies exclusively in the $u_{x}-u_{z}$ plane, since the Lorentz force and dominant motion are in the $x-z$ plane, and radiation friction damps any motion perpendicular to the plane. Figure 3(a) shows the variation of the longitudinal velocity with time, and Fig. 3(b) plots the resultant escape angle against time elapsed. The stochasticity in the motion is caused by the evolution of $v_{r}(t)$ (not shown here); the exit angle follows a clear trend. In the presence of multiple electrons, we expect the stochastic effects to "cancel" out. What is clear is that some form of control can be performed for the individual particle too.

The trends predicted by our heuristic argument, Eq. (2), are quite closely followed for trapped particles in Fig. 2, but not in the case of linearly polarized lasers in Fig. 3. Numerical simulations in the following sections delightfully demonstrate that this deviation seems to cancel out if we start with a distribution of several particles over a sizable region. It also turns out that one may not need to vary the frequencies of the laser pulse adiabatically to achieve analytically tractable results when considering a distribution of particles. It is encouraging to learn, in the following sections, how the use of fixed frequencies, which can be selected from among a few discrete values, works perfectly well. In the ZMF, the final average exit angle is quite robust to changes in the initial conditions of the seeds. Thus we can anticipate the central feature of attractor dragging from classical motion to be qualitatively true also in the QED regime, at least for planar laser pulses. For focused Gaussian laser pulses, too, we shall see our results being statistically accurate even though the attractor-dragging argument is not strictly valid due to the spatiotemporal inhomogeneity of the laser pulse.

\section{MONTE CARLO SIMULATIONS}

For the Monte Carlo simulations, we use the technique proposed in Ref. [50] and write a code that is publicly available at a GitHub repository [51]. We evolve all three components of momentum and position of electrons and positrons using the Lorentz force, neglecting interparticle interactions. To any particle, at the beginning, we assign an optical depth $\tau=0$. Choosing an exponentially distributed (with mean 1) random positive real $\tau_{f}$, we evolve $\tau(t)$ as $d \tau / d t=h\left(\chi_{e}\right)$ until it hits $\tau_{f}$. Here, $h\left(\chi_{e}\right)$ is obtained from the quantum synchrotron emissivity $F\left(\chi_{e}, \chi_{\gamma}\right)[23,50]$ and can be tabulated for efficiency [52]. At this point, $\chi_{\gamma}$ for the emitted photon is randomly sampled from the distribution $\mathcal{D}\left(\chi_{\gamma}\right) \sim F\left(\chi_{e}, \chi_{\gamma}\right) / \chi_{\gamma}$. The direction of propagation is assumed to be parallel to the direction of motion of the original electron or positron; the energy is given by $2 \chi_{\gamma} \gamma / \chi_{e}$, using, again, $\gamma$ and $\chi_{e}$ for the electron and positron at the moment of photon emission. The momenta are updated accordingly, and $\tau$ for the particle is reset to 0 . Production of $e^{-} e^{+}$pairs from photons uses a very similar procedure, with pairs emitted along the photon's direction of motion [53]. First, consider the case with $a_{2} / a_{1}=\lambda=1$. We start out with initial positions randomly, uniformly distributed near the origin and a Maxwell-Jüttner (MJ) momentum distribution with temperature (normalized to $\left.m_{e} c^{2}\right) T \sim(0.01-0.1)$. The approximation of Maxwell-Jüttner thermal distribution is reasonable since the seeded electrons arise from the ionization of ambient air present in the experiment. For the intensities considered here, these tunnel-ionized electrons will acquire the Maxwell-Jüttner distribution rather easily in a fraction of the laser period. It may be noted, however, that the precise choice of initial distribution (single electron, cold, random-uniform, thermal MJ distributions) was found to be of little consequence for the results. This is also consistent with what is expected of avalanche-type cascades [12]. However, if one were to consider the interaction of monoenergetic electron beams from laser-wakefield accelerators, such as envisaged in Refs. [24,25], then deviations from the results shown here can be expected.

We run each of our simulations thrice to obtain error estimates for points in our plots. Generally, we have used error bars extending up and down a single standard deviation. We begin with $a=a_{0}=450$ and 100 electrons, integrating over $20 \pi / \sqrt{\omega_{1} \omega_{2}}$ time, and plot the average $x$-velocity of the electrons in Fig. 4, along with the velocity of the ZMF, as given by 


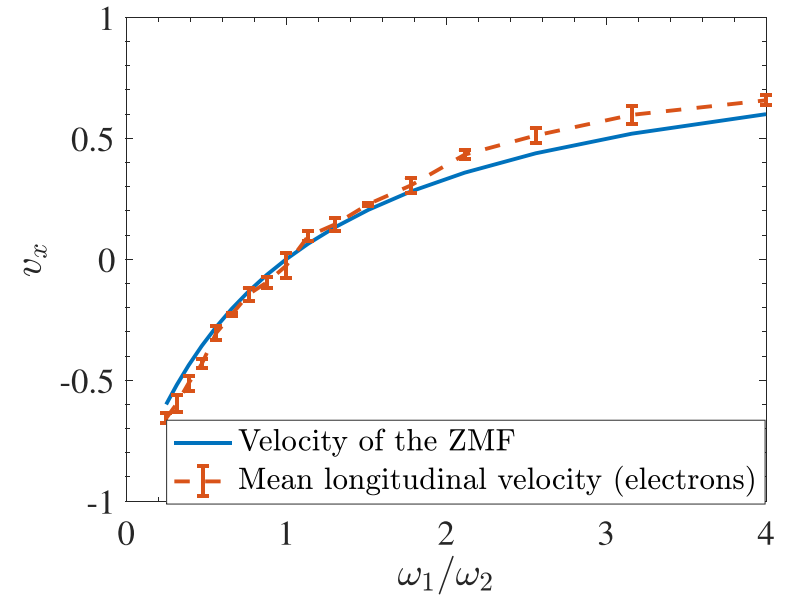

FIG. 4. $v_{\mathrm{ZMF}}$, and the mean longitudinal velocity of electrons vs time in linearly polarized pulses. The geometric mean of wavelengths is fixed at $1.1 \lambda_{L}$. Other parameters are $\lambda=1$ and $a=450$.

$v_{\mathrm{ZMF}}=\left(\omega_{1}-\omega_{2}\right) /\left(\omega_{1}+\omega_{2}\right)$. The accuracy of this heuristic estimate is remarkable.

Figure 5(a) shows the mean angle of escape of electrons, positrons, and photons, computed, given a probability distribution function for a certain particle to have an exit angle $\theta, P(\theta)$, as $\left\langle\theta_{E}^{\text {esc }}\right\rangle=\int_{-\pi / 2}^{\pi / 2} P(\theta) \theta d \theta$, along with the mean angle of emission of energy, computed as $\left\langle\theta_{E}^{\text {ener }}\right\rangle=\int_{-\pi / 2}^{\pi / 2} \sqrt{u^{2}+1} P(\theta) \theta d \theta$, using Eq. (2). Electrons and positrons are dragged along by the ZMF due to electromagnetic forces, so their curves closely match the heuristic result. In comparison, the mean-energy-emission-angle curves have an upward bias when compared with the mean-particleexit-angle curves: Particles which have been accelerated for a longer time (i.e., older particles) are more energetic — but by virtue of being accelerated longer, they also travel closer to the $x$ axis and thereby bias the curve. Photons, meanwhile, are not dragged along with the ZMF by themselves; they are merely released by accelerated electrons and positrons. Many of these photons are released in the earlier stages of $e-p$ acceleration; this lowers the mean exit angle for photons below that of other particles. However, since photons released in these early stages have considerably lower energy than those released by faster electrons later in the cascade, the mean photon energy emission angle stays close to the electron- and positron-exitangle curves. The transverse bias of the angular spectrum hints that we are dealing with avalanche-type cascades [12]. Figure 5(b) shows the spread of the jets of particles (and the carried energy), as measured using the standard deviation as $\theta_{S}^{\text {esc }}=\left(\left\langle\theta_{E}^{\text {esc } 2}\right\rangle-\left\langle\theta_{E}^{\text {esc }}\right\rangle^{2}\right)^{1 / 2}$ and $\theta_{S}^{\text {ener }}=\left(\left\langle\theta_{E}^{\text {ener } 2}\right\rangle-\left\langle\theta_{E}^{\text {ener }}\right\rangle^{2}\right)^{1 / 2}$. Note the minimum at unity for electrons and positrons and the maximum for photons. In general, moving faster relative to the ZMF should make the jets appear narrower; this is indeed true for frequency ratios significantly deviating from unity. However, the minimum at unity seems to be associated with phase space contraction of the attractor [31], the effects of which should be especially prominent and lead to narrowing for frequency and amplitude ratios close to 1 . Also, as the upper and lower halves of the pair jet bend and overlap, the jet spread can artificially appear to increase until jet narrow-

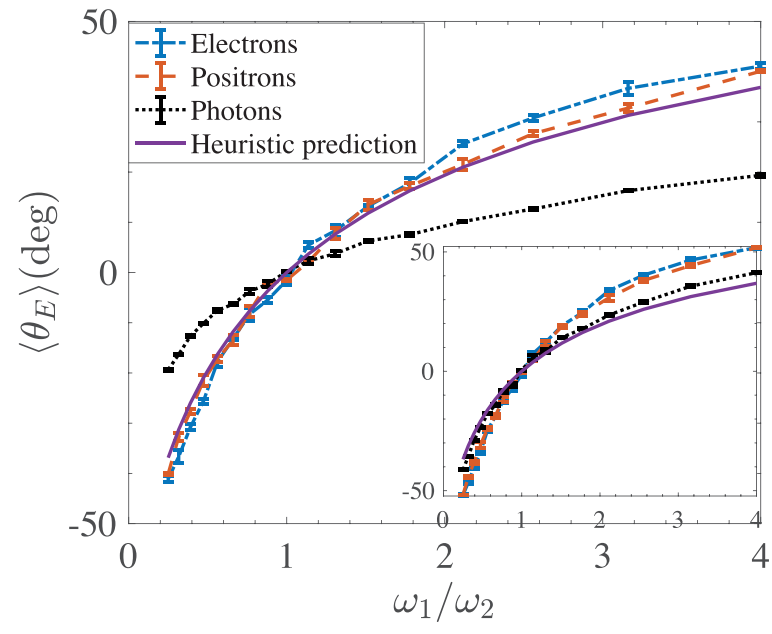

(a)

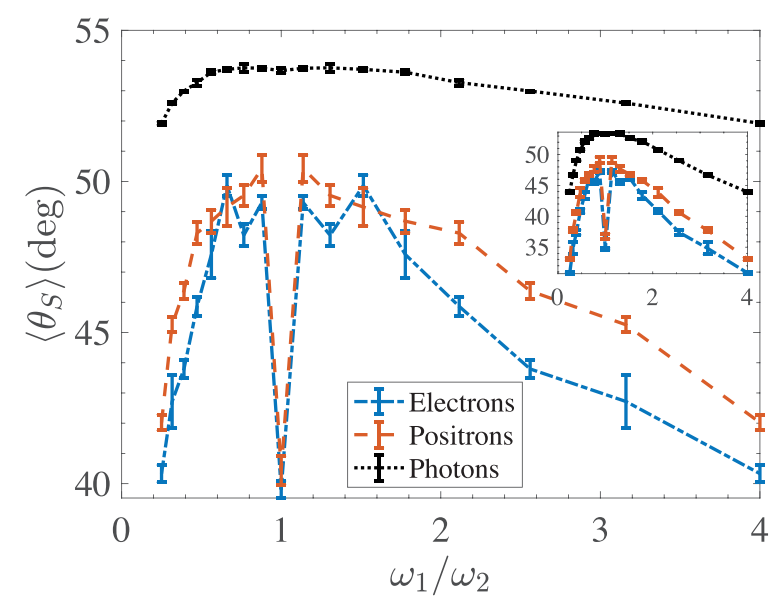

(b)

FIG. 5. (a) The mean angle of emergence of particles and the energy they carry (inset), as measured from the transverse plane, along with our heuristic prediction using Eq. (2). (b) Spread and energy distribution (inset) carried by them, as measured using the standard deviation. Other parameters are $\lambda=1$ and $a=450$ (LP pulses) with the product of wavelengths fixed at $\approx 1.21 \lambda_{L}^{2}$.

ing due to relativistic motion dominates at larger frequency ratios. The bottom graphic of Fig. 1 schematically depicts this overlap. It summarizes the frequency-ratio-dependent properties of fermion jets that we have observed in the numerical simulations.

Note how $\left\langle\theta_{S}^{\text {ener }}\right\rangle\left\langle\left\langle\theta_{S}^{\text {esc }}\right\rangle\right.$ for each particle. This indicates that the particles with greater energy are sent at narrower exit angles, closer to the mean, as should well be the case. Particles that deviate feel larger radiation pressure from light beams impinging on them preferentially in their rest frame, on average. This narrows the energy spread, as compared with the particle spread. Since we start off with electrons, these are accelerated for a slightly longer time than positrons (which are generated only out of pair production) and thus come out at higher energies, closer to the mean, in slightly narrower jets, than positrons do. Meanwhile, for photons, which are 


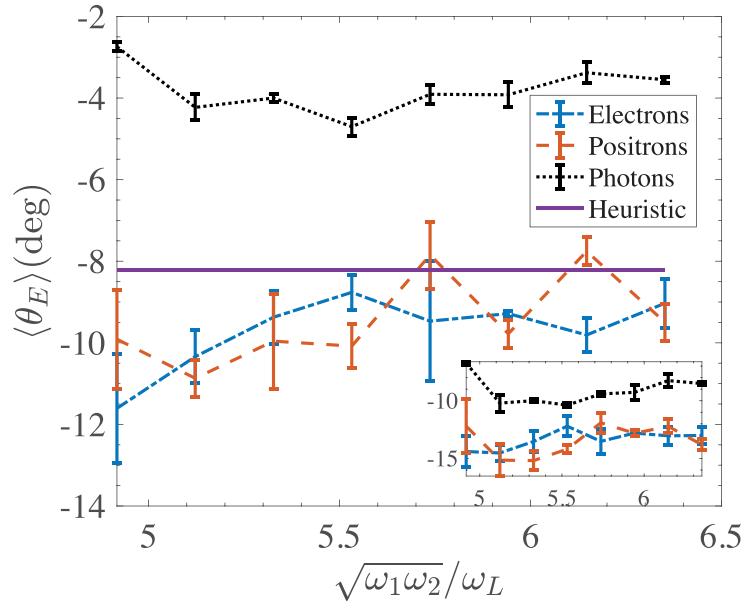

(a)

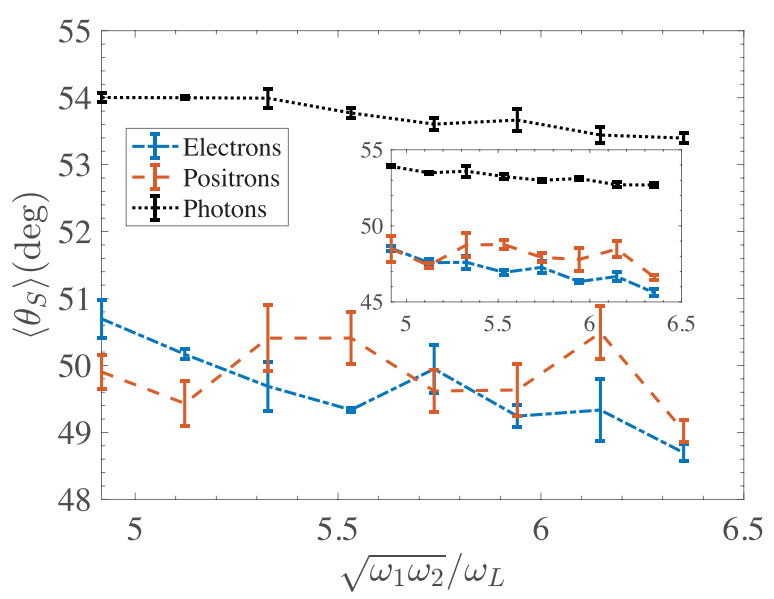

(b)

FIG. 6. (a) Mean emergence angle of particles and their energy (inset), and heuristic prediction. (b) Spread of particles and energy distribution (inset) for motion in LP laser pulses with $\lambda=0.8$ and $a_{0}=450$ and frequency ratio fixed at $4 / 3$.

not accelerated by the fields, the spread is biased towards the higher side by those released by low-energy particles at the beginning of the cascade. Figures 6(a) and 6(b) show the mean exit angle and spread for particles and the energy they carry, with frequencies varying while maintaining a constant ratio. Trends only indicate a weak increase in the mean exit angles, and weak decrease in the spread of the beam, with frequencies; they are barely noticeable. This implies that at higher frequencies, beams get narrower, albeit not very significantly. Independence or weak dependence of exit angle and spread from individual frequencies bolsters our heuristic results. It establishes that jet properties depend strongly only on the ratio of frequencies and not on their individual values.

Next, we look at the effect of varying amplitudes (the ratio, $\lambda$, or $a_{0}$ itself) while keeping frequencies fixed. Varying $a_{0}$ is computationally expensive (the number of produced particles blows up exponentially as the field increases) and thus has been studied only with 2D SMILEI simulations. It does not produce interesting trends. However, this exponential cost does indicate that we are likely dealing with avalanche-type

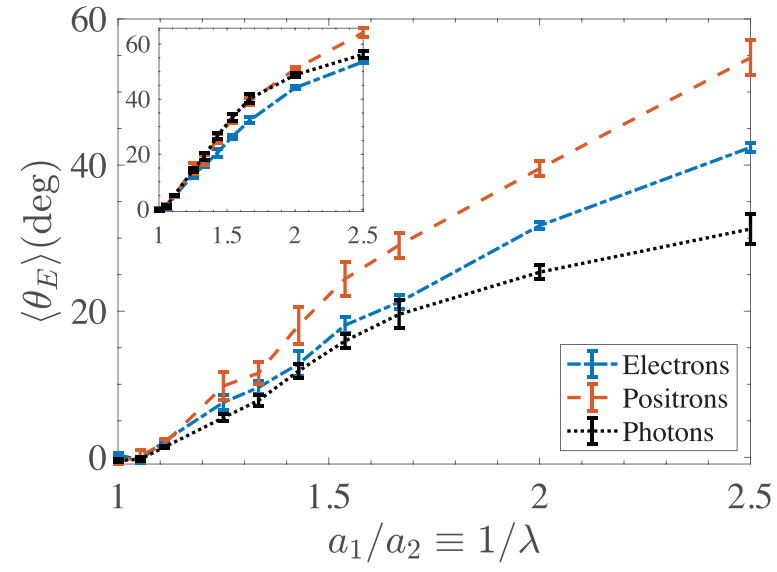

(a)

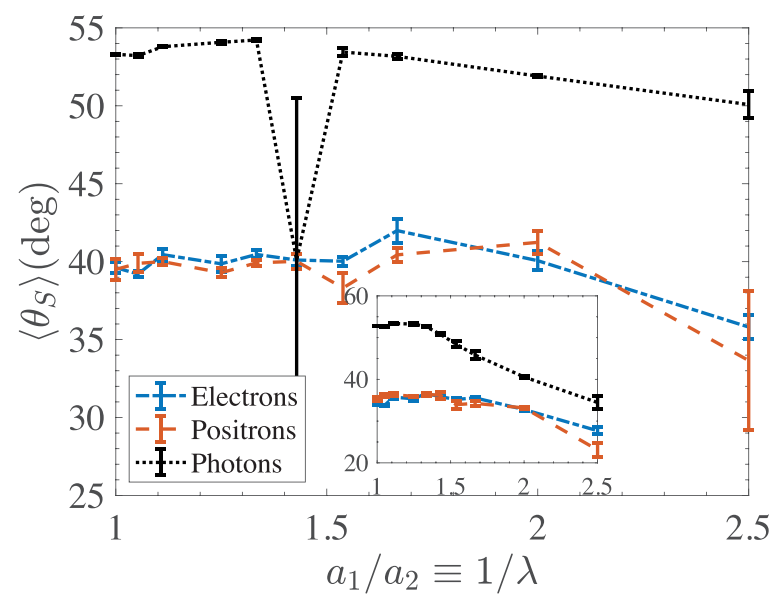

(b)

FIG. 7. (a) Mean exit angles of particles and energy carried (inset). (b) Spread and energy distribution (inset). Motion in LP laser pulses with $\omega_{1}=\omega_{2}=6.4 \omega_{L}$ and $a_{1}=450$.

cascades [12]. Therefore, in Fig. 7, we held $a_{0}=450$ and $\omega_{1}=\omega_{2}=6.4 \omega_{L}$. Figure 7(a) shows the mean exit angle varying with amplitude ratio. The wave can be seen as the superposition of a standing wave and a pure wave propagating to the right; the latter causes acceleration in the $+x$ direction as the ratio deviates from 1 . The spread of the wave decreases as the acceleration picks up, again, as shown in Fig. 7(b). Reasons why the curves are positioned the way they are directly carry over from the frequency ratio argument.

It may be pertinent to see the actual probability distributions that we have been interested in. Figure 8(a) shows the distribution of angles for $\omega_{1}<\omega_{2}$. Note the bias towards the negative angles. In the same situation, look at Fig. 8(b), which shows the energy distribution histogram for electrons and positrons. As expected, they peak at $\gamma \sim a_{0} \sim 10^{2}$. Our integration time (ten wave periods in the ZMF, $20 \pi / \sqrt{\omega_{1} \omega_{2}}$ ) was quite arbitrarily chosen. Figure 8(c) shows the variation of exit angle and angular spread of the jet, demonstrating stabilization with time. This hints towards ergodicity and mixing being maintained in this process. Finally, Fig. 8(d) shows the angular distribution of positrons and their energy, establishing that the particles traveling near the "center" (mean or mode) 


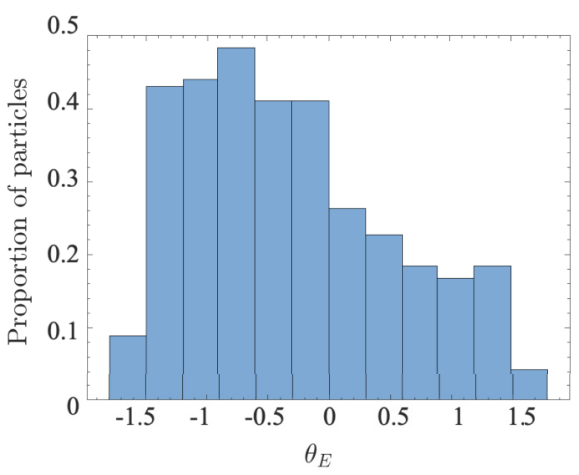

(a)

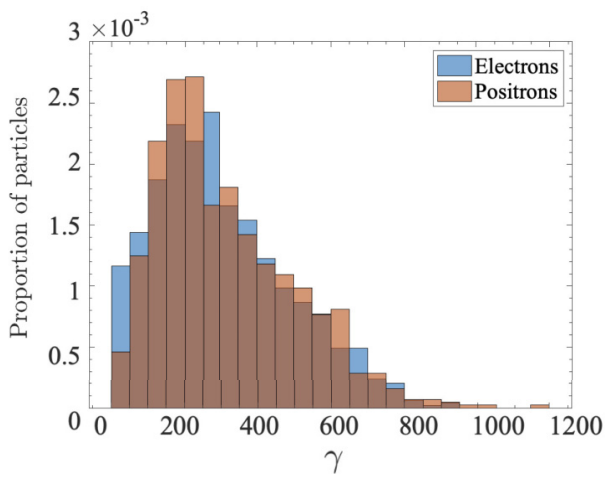

(b)

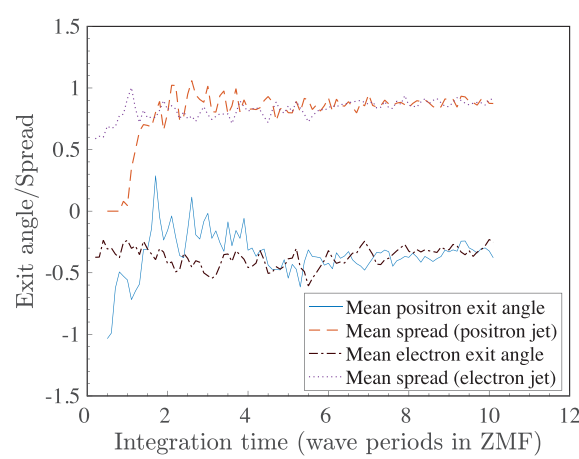

(c)

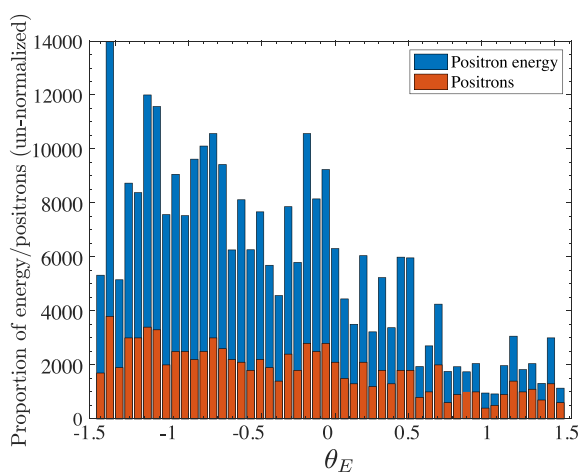

(d)

FIG. 8. Probability distribution of quantities in the cascade. (a) Electron exit angle, with $a_{0}=450, a_{1}=a_{2}, \omega_{1}=4.3 \omega_{L}$, and $\omega_{1} / \omega_{2} \approx 0.57$. (b) Energy distribution. (c) The mean exit angle (and spread) for electron and positron jets. (d) Distributions of positrons and their energy. All angles are in radians.

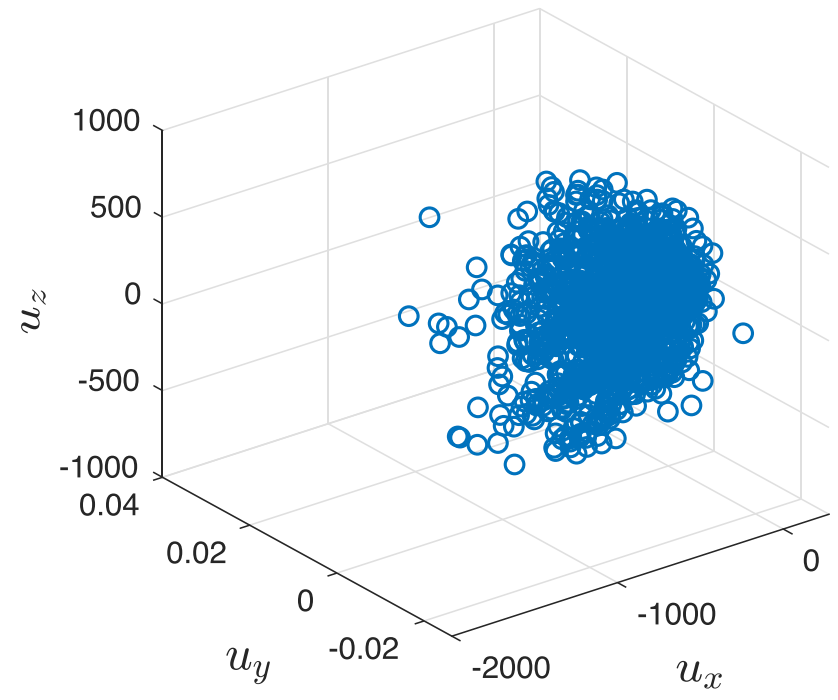

(a)

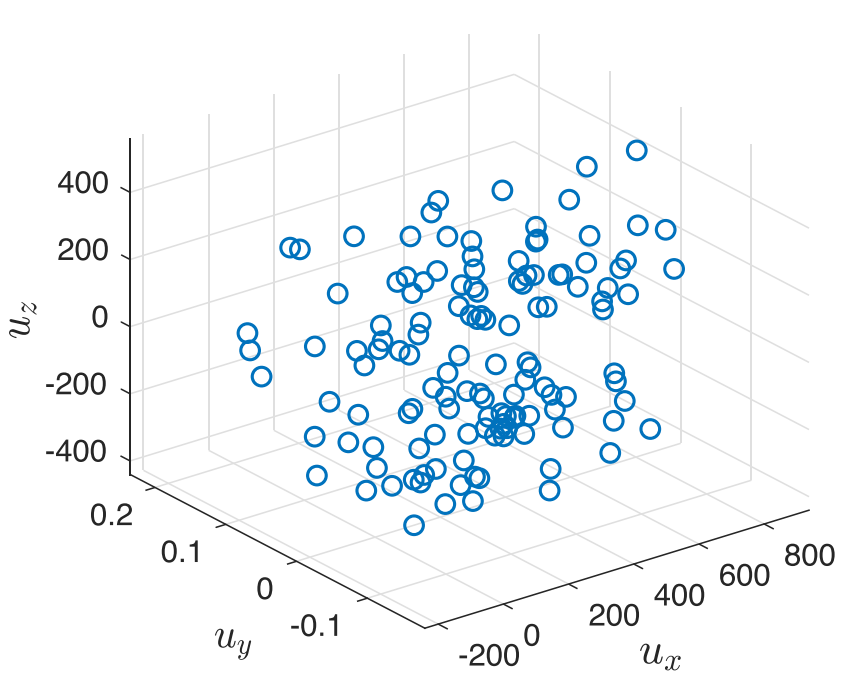

(b)

FIG. 9. Momentum-space distribution of particles. (a) Same parameters as in Fig. 5, with $\omega_{1} / \omega_{2}=1 / 4$. Note the tiny scale on the $y$ axis: Motion is essentially thermal in that direction. (b) With the same parameters as in Fig. 7, and $a_{2} / a_{1}=0.4$.

of the beam carry the most energy, as surmised from earlier figures.

Under the plane wave approximation $\left(r_{0} \gg \lambda_{L}\right)$ used throughout this section, it is worth noting that accelerated particles are concentrated in the $x-z$ plane. There is no acceleration perpendicular to the $x-z$ plane. Photons and pairs are, in turn, released in the dominant direction of motion of the existent particles: the $x-z$ plane. We might thus expect the jet to be tightly pancaked in that plane, perpendicular to the magnetic field direction. Indeed, refer to the scales of the axes in Fig. 9, which shows the distribution of particles in momentum space for the cases discussed in Figs. 5 and 7. The stretch of the $u_{y}$ axis is smaller than any of the other axes. 
This observation may not be true for focused Gaussian laser beams. The magnetic field is no longer strictly perpendicular to the electric field and has an axial component [54], causing the particles to accelerate out of the plane with relativistic velocities. This can complicate the distinction between cascade and vacuum-decay products discussed later in Sec. VI.

\section{2D PIC SIMULATIONS}

We now validate our previous predictions by carrying out two-dimensional PIC simulations using the open-source code SMILEI [55]. Our objective in doing PIC simulations is not to study the plasma effects, but to verify the results from the simplified and more efficient Monte Carlo simulations used in the previous section and test our results with the laser pulses that are experimentally viable. We first use the linearly polarized plane laser pulses to validate our results from Sec. IV, albeit with pulses of finite duration, the temporal profiles being Gaussian, demonstrating that the results obtained in the previous section are robust to the use of finite-sized pulses. These plane laser pulses have Gaussian temporal profiles with duration $20 \lambda_{L} / c$ and a full width at half maximum (FWHM) of $10 \lambda_{L} / c$. Afterwards, we employ laser pulses with Gaussian spatiotemporal profiles. For this part, we shall use lasers of spot size $r_{0}=5 \lambda_{L}$. Furthermore, to account for the possible misalignment errors in experiments, we offset the laser pulses' focus positions by $\Delta x=0.1 \lambda_{L}$ along both axes. The simulation region has a length $L_{x}=20 \lambda_{L}$ along the $x$ axis and $L_{y}=0.5 \lambda_{L}$ along the $y$ axis. The step sizes in the $x$ and $y$ directions are $\delta x=0.065 \lambda_{L}$ and $\delta y=0.13 \lambda_{L}$, respectively. Absorbing boundary conditions on the $x$ axis and periodic boundary conditions on the $y$ axis are used. The initial seed density, $n_{s}=10 / \lambda_{L}^{3} \approx 10^{13} \mathrm{~cm}^{-3}$, is concentrated in a $4 \lambda_{l}$-thick strip about the center of the simulation region and composed of only cold electrons. The initial plasma frequency is thus $\omega_{p} \approx 10^{-4} \omega_{L}$.

The stochastic model of photon emission and pair generation by the Breit-Wheeler process in SMILEI employs a fully quantum Monte Carlo model [52,53]. For calculating the photon and pair productions in PIC codes, certain assumptions are needed: Motion of the particle is ultrarelativistic, electromagnetic fields are smaller than the critical Schwinger field and vary slowly over the formation time of the emitted photon, and particles radiate independently of their neighbors. Under these assumptions, the emission of photons and pair creation can be described by the process of nonlinear inverse Compton scattering [26]. These assumptions are always satisfied in the PIC simulations carried out. For numerical implementation in SMILEI, photon emission and pair creation are assumed to be random-walk processes. The numerical procedure to calculate the photon and pair generation is essentially the same as in Sec. IV. In the beginning, initial and final optical depths (between 0 and 1) are assigned to a photon. The optical depth evolves with time as the particles propagate in the laser field. When the final optical depth is reached, a photon is emitted. The time evolution of the optical depth is equal to the production rate of pairs [53]. The value of $\chi$ parameters of emitted particles can be obtained by inverting the cumulative probability distribution function of the respective species [21,50]. Unlike our Monte Carlo simulations, which

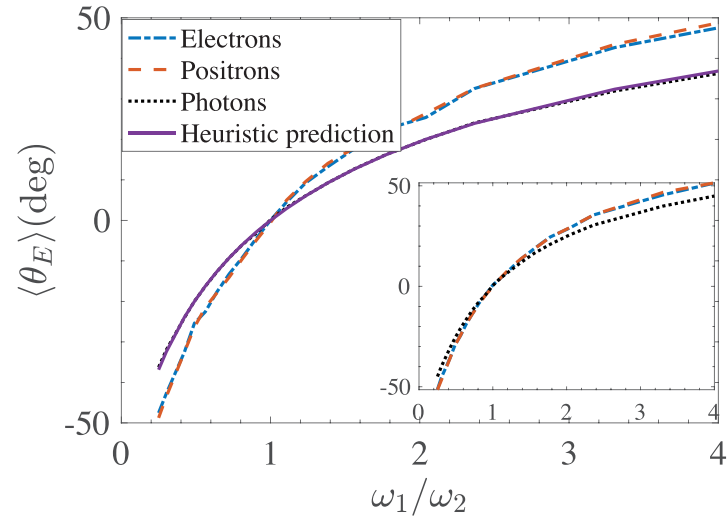

(a)

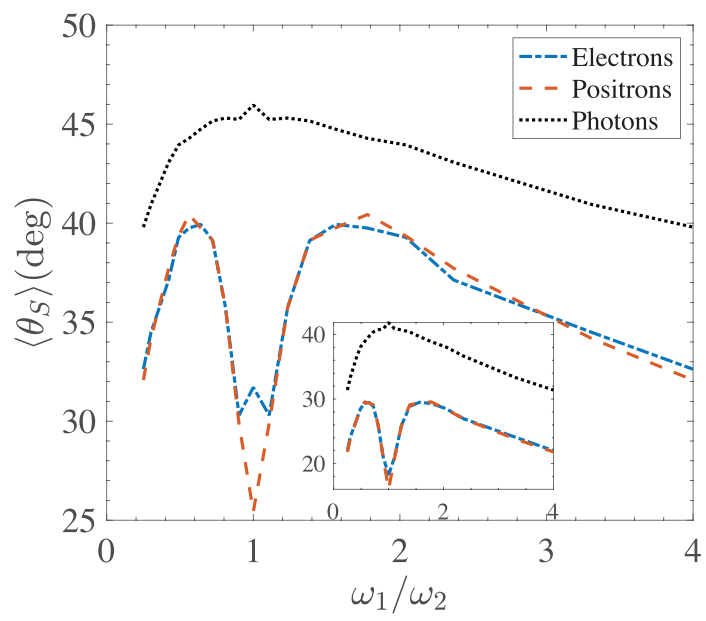

(b)

FIG. 10. Mean exit angle (a) and spread (b) for jet particles (insets: the energy carried by particles) for fixed product of wavelengths $\left(1 \mu \mathrm{m}^{2}\right)$, and varying frequency ratio for $a_{0}=1400$. Note the similarity to corresponding Fig. 5.

advance individual particles in an independent background field, SMILEI numerically solves the Vlasov-Maxwell system of equations using a PIC algorithm. The numbers of pairs generated at the simulations have density that is similar to or higher than the initial density, $1.5 \times 10^{13} \mathrm{~cm}^{-3}$. However, these pairs are dispersed over the whole simulations box. Thus the plasma density is still too small to show any collective plasma effects in the interaction with the laser. As before, the dynamics and statistics indicate an avalanche-type cascade [12].

Figure 10 shows excellent agreement with Fig. 5. The values of the spread, in general, however, are smaller in the $2 \mathrm{D}$ scenario than in $3 \mathrm{D}$, likely due to effects we did not account for in the 3D case, such as the Gaussian profile of the laser pulse. As before, unlike the photon spread curve, the photon energy spread curve closely follows the electron and positron spread curves, since it is biased by the highly energetic photons released by electrons and positrons later in the cascade. Figure 11 presents the PIC simulation results of Fig. 6. Figure 11(a) is a flipped version of Fig. 6(a) (simply since we used $\lambda=1.2>1$, unlike $\lambda=0.8$ used before), and 


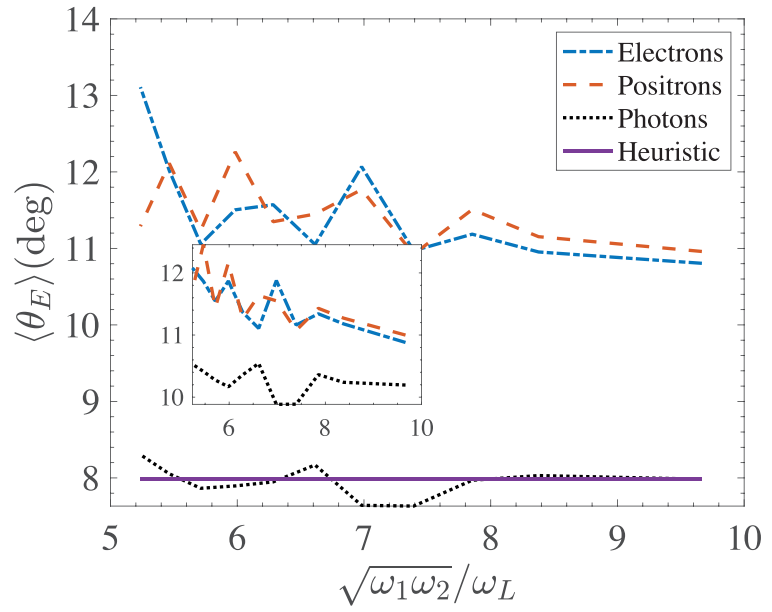

(a)

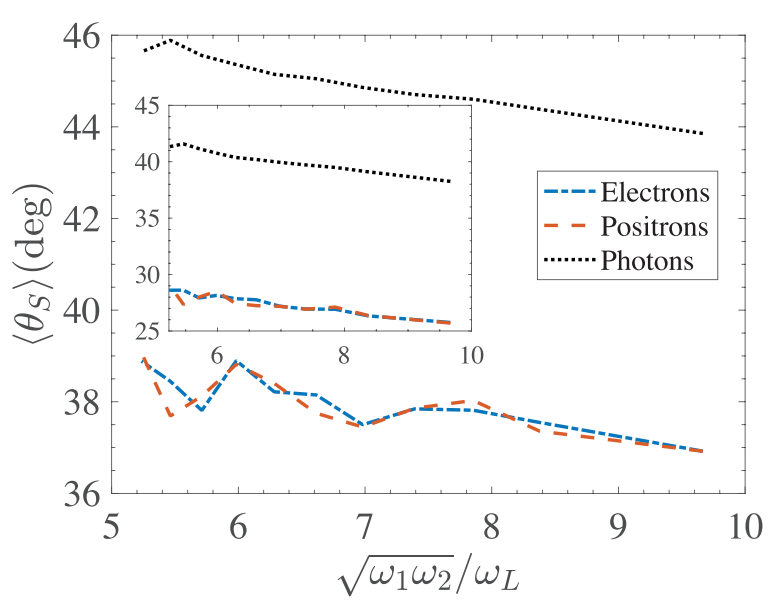

(b)

FIG. 11. Mean exit angle (a) and spread (b) (insets: energy carried by particles) for varying frequencies with fixed ratio at 1.32 and $a_{0}=1400$. Note the similarity to corresponding Fig. 6 .

Fig. 6(b) also closely follows Fig. 11(b). In Figs. 12 and 7, the range is different from that used earlier, but the behavior of curves is, again, quite the same. In Fig. 12(a), we obtain a naive estimate of the exit angle by postulating that in the frame where the exit angle is $0,\left\langle v_{x}\right\rangle=0$ if and only if radiation pressure is equal from both waves if and only if $\omega_{1} / \omega_{2}=\lambda$ (Poynting vector vanishes), and proceeding to find the exit angle, as before. However, this simplistic argument does not give a good approximation. It does, however, follow from the vanishing-power-flow argument we shall present in Sec. VI.

Figures 13(a) and 13(b) show curves we could not capture with our Monte Carlo simulations in Sec. IV, due to computational limitations (pair production blows up exponentially with increasing amplitude, indicative of an avalanche [12]). In Fig. 13(a), plotted at equal frequencies and a constant amplitude ratio, all the curves show a decrease (significant for photons) as the parameter $a_{0}$ is increased and gradually settle and converge; that is, as electromagnetic forces grow with the amplitude, the system appears to come to a steadier state, given a constant integration time. This makes sense;

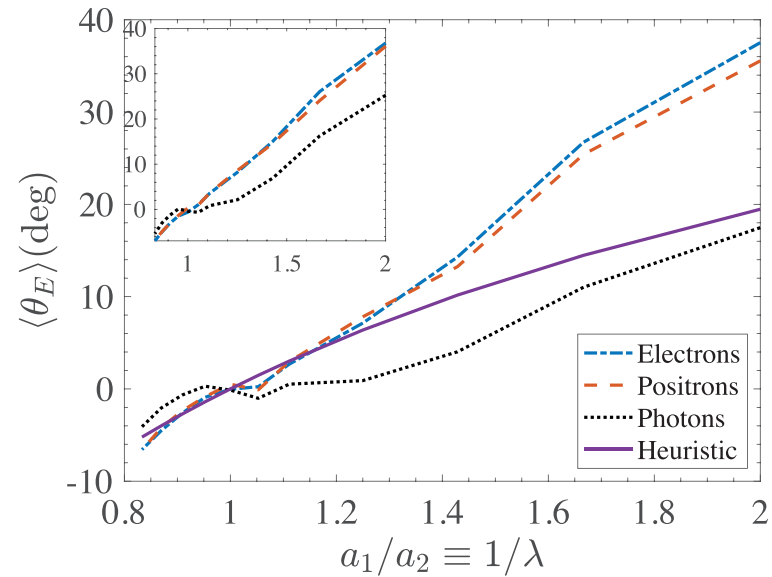

(a)

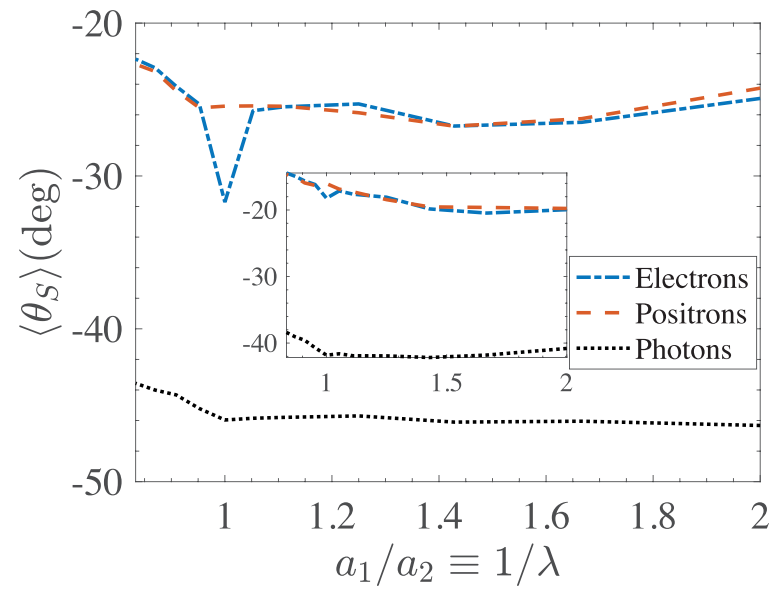

(b)

FIG. 12. Mean exit angle (a) and spread (b) (insets: energy carried by particles) for varying amplitude ratio, while holding $a_{0}=$ 1400. Note the similarity to corresponding Fig. 7. Please note that in (b), we have plotted the negative spread for convenience in positioning the inset [this was also done in Fig. 16(d)].

stronger forces cause faster thermalization and stabilization. The mean exit angle for photons differs significantly from that for electrons and positrons for lower amplitudes and a given integration time, but the difference is much lessened for higher amplitudes, as the graph shows. Again, due to computational limitations, we could not obtain data beyond $a_{0}=1800$. The spread of the jets shows a slow decrease with increase in $a_{0}$, in Fig. 13(b). With the shown range, it may be premature to comment on convergence here.

Lastly, some distributions may be of interest. In Fig. 14 we move with parameters used in Figs. 10(a) and 10(b), with a frequency ratio of 0.8. Figures 14(a) and 14(b) show the distribution of particles and their energy against exit angle. Note the bias to the left, since $\omega_{1}<\omega_{2}$. Again, it is visible that particles closest to the "center" of the respective beam carry the most energy and therefore narrow the energy distribution in comparison with the particle distribution. The structure of the momentum-space plots is particularly elegant when viewed at higher resolutions, and we include it here (Fig. 15) 


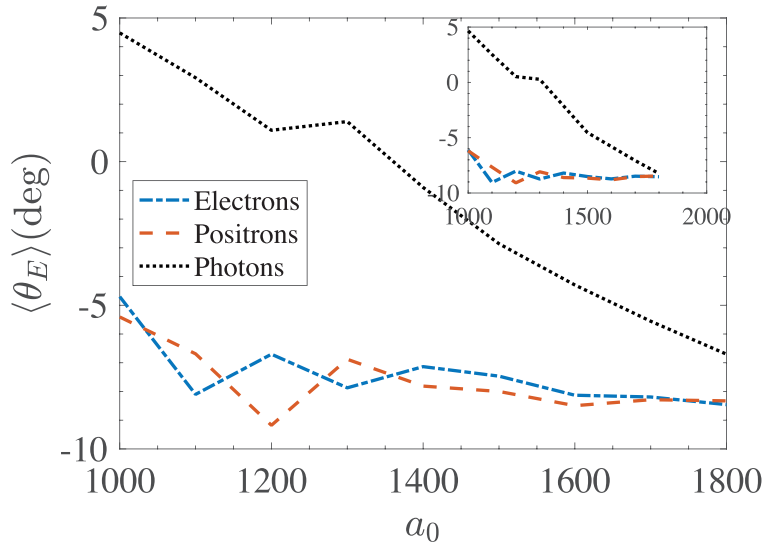

(a)

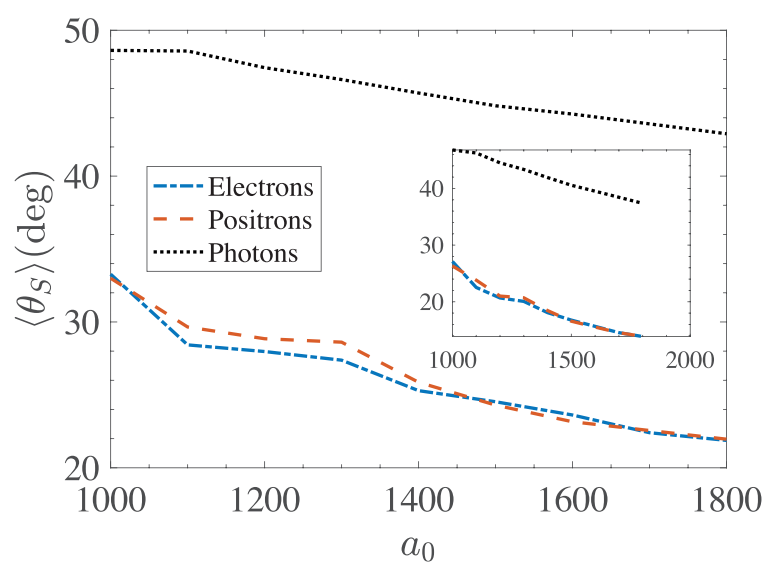

(b)

FIG. 13. Mean exit angle (a) and spread (b) for jet particles (insets: energy carried by the particles) for $a_{2} / a_{1}=1.25$, with $a_{1} \equiv$ $a_{0} / 2$. Similar to corresponding Fig. 6 .

for an appropriate set of parameters. Note the bias towards the left since $\lambda=1.25$.

What we can conclude, however, is that it is entirely possible to control exit angles and spreads of jets by varying the amplitude and frequency ratios of colliding laser pulses. Moreover, the dependence of properties of these jets on the individual values of amplitudes and frequencies is only weak. One could write an expression for an estimate for the combined dependence of the exit angle on the frequency and amplitude ratios. If we take our rationale for moving to the zero-power-flow frame for the case of varying amplitude ratios a step further, we can simply write $\left\langle v_{x}\right\rangle \equiv\left(\omega_{1} a_{1}-\right.$ $\left.\omega_{2} a_{2}\right) /\left(\omega_{1} a_{1}+\omega_{2} a_{2}\right)$. Following Chap. 4 of Ref. [56], this can be stated with greater clarity: For generally oriented electric and magnetic fields $\mathbf{E}$ and $\mathbf{B}$, i.e., for any number of lasers intersecting at arbitrary angles with arbitrary amplitudes and frequencies, the frame where the Poynting vector vanishes, the ZMF for equal amplitudes or zero-average-power-flow frame (ZPF) in general, has a rapidity of $\alpha$ and moves parallel to the Poynting vector in the laboratory frame, where tanh $2 \alpha=$ $2 \mathbf{E} \times \mathbf{B} /\left(\mathbf{E}^{2}+\mathbf{B}^{2}\right)$, of which one can verify that our result is a special case.

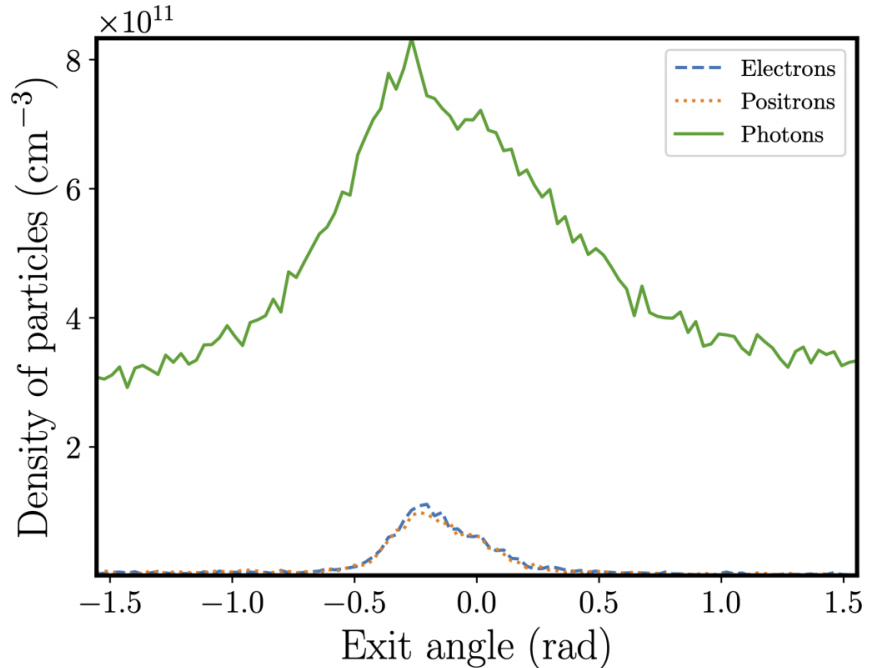

(a)

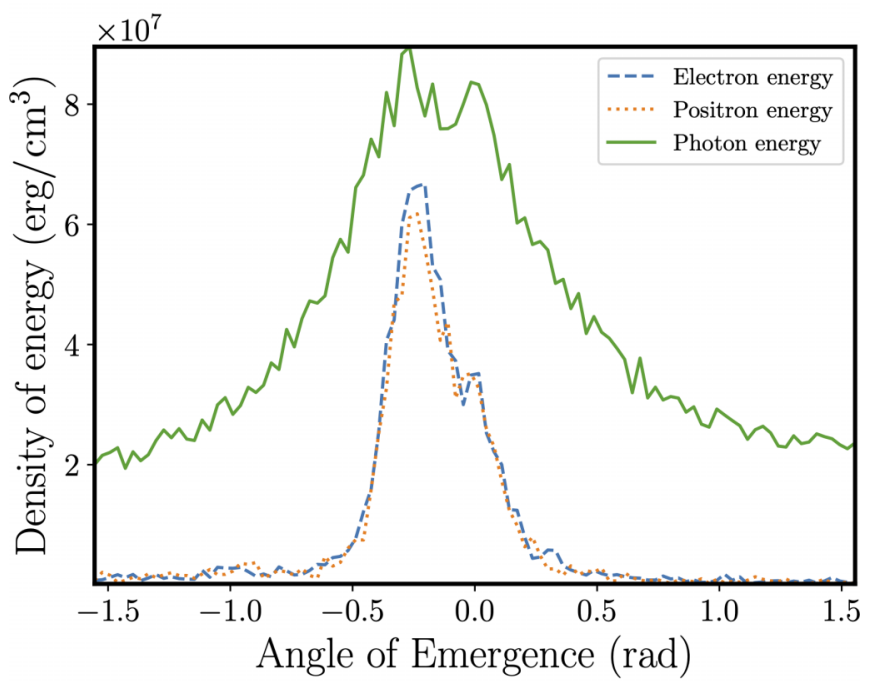

(b)

FIG. 14. (a) Distribution of particles for varying exit angles. (b) Distribution of energy carried by the same particles. Note that the energy is narrowly distributed, as in Monte Carlo simulations. The values are sampled at $t=138.74 / \omega_{L}$. Other parameters are the same as in Figs. 12(a) and 12(b), with $\omega_{1} / \omega_{2}=0.8$.

We conclude this section by presenting some of the results for focused Gaussian laser pulses in Fig. 16. On comparing with the results in Figs. 10 and 12, we find similar trends, validating the robustness of our results. The other plots are not shown for this case, but they also depict similar trends for spatially planar lasers with Gaussian temporal profiles shown in Figs. 11 and 13 earlier.

\section{COMPARISON WITH JETS FROM THE VACUUM DECAY}

The production statistics of $e-p$ pairs by strong laser pulses (several orders of magnitude stronger than those used in this paper) in the absence of seed electrons or positrons was studied in Ref. [7]. The statistics immediately after vacuum decay 


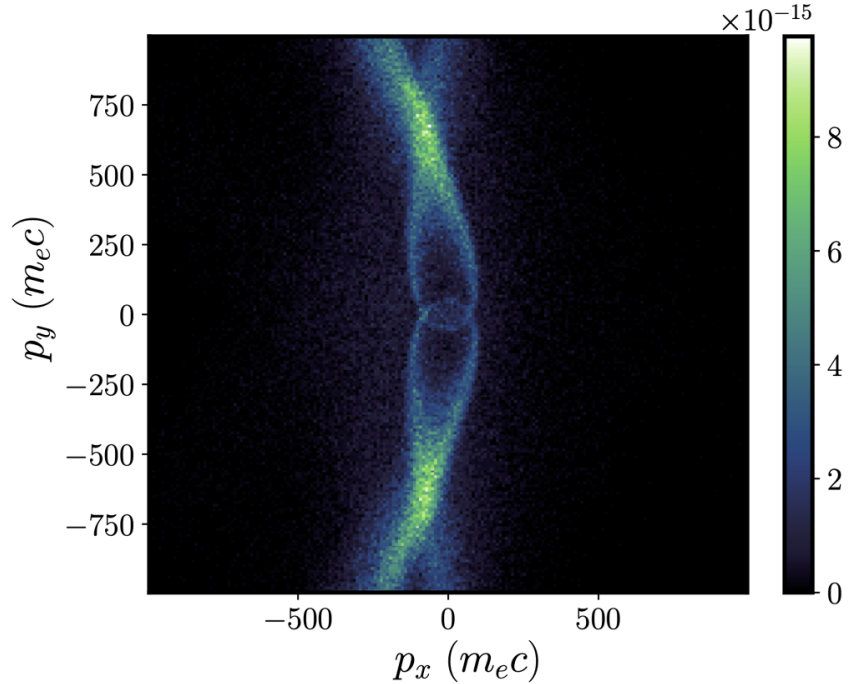

(a)

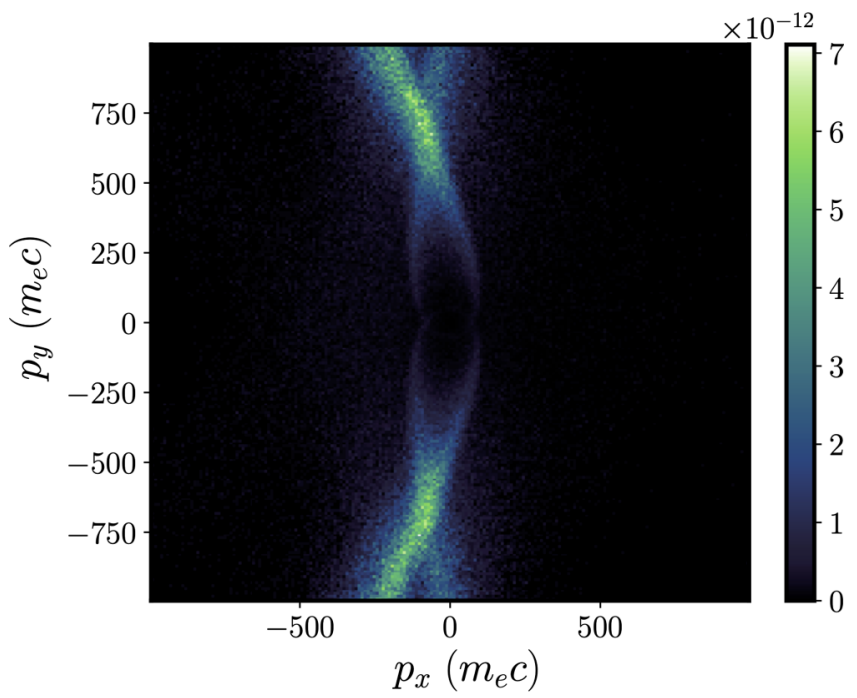

(b)

FIG. 15. (a) Electron number density in $\left(p_{x}-p_{y}\right)$ space and (b) electron energy density in $\left(p_{x}-p_{y}\right)$ space, in $d p_{x} d p_{y}$ volume element at a time $138.74 / \omega_{L}$, using $\lambda=a_{2} / a_{1}=1.25$, with wavelengths maintained at $\lambda_{L} ; a_{0}=1800$.

were analyzed analytically, and it was argued that particles are produced with zero momentum parallel to the electric field and move off (initially) collimated tightly in planes perpendicular to the electric field direction. Thus the cascade products are confined to the electric field plane. The vacuum-produced pairs were symmetrically distributed in a frame where the Poynting vector $\mathbf{S}=0$ : the ZPF (ZMF for equal amplitudes), moving parallel to $\mathbf{S}$. This directionality of the emitted jets can enable distinguishing between the vacuum-decay and cascade products. A visual depiction of the vacuum-decay jet, and its relationship with the cascade jet, is shown schematically in Fig. 1. It must be stressed at this point that this difference between spectra (collimation plane) for cascade and vacuumdecay products is prominent only when using laser pulses with large spot sizes and during the early stage of the vacuum decay. For tightly focused laser pulses, the vacuum-decay

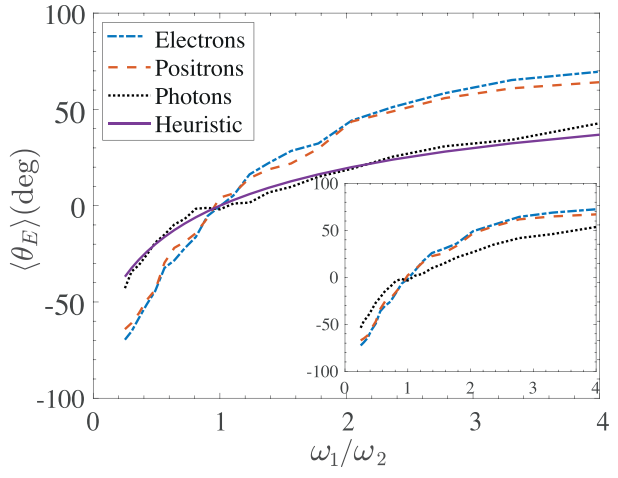

(a)

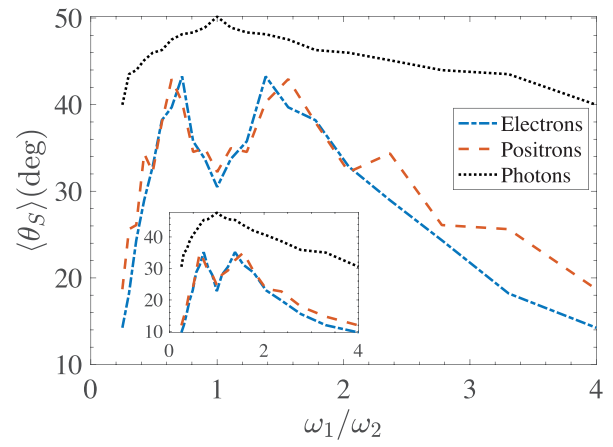

(b)

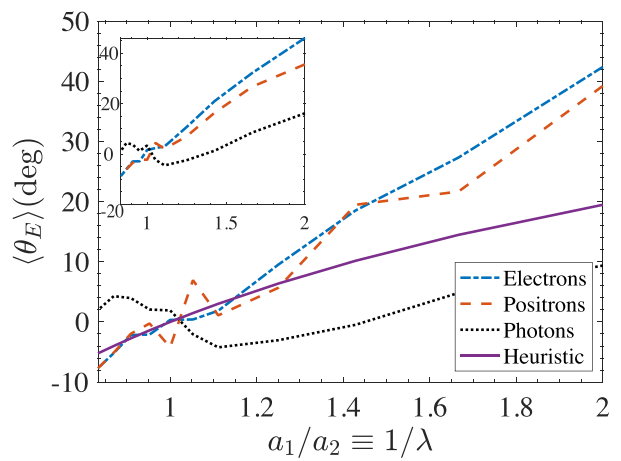

(c)

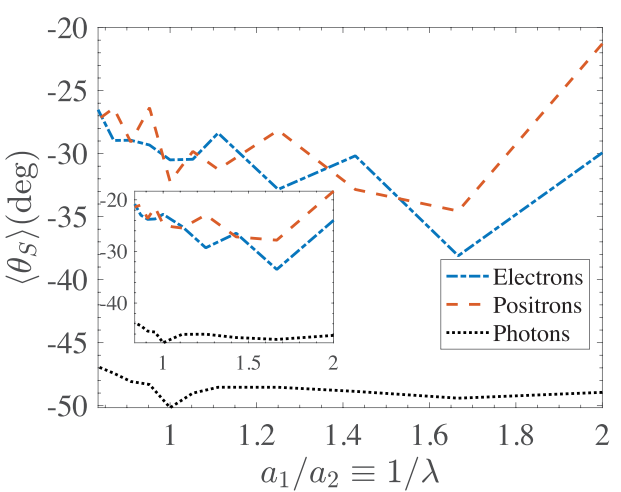

(d)

FIG. 16. Mean exit angle and spread in the case of laser beams with Gaussian spatiotemporal profiles having an offset (energy distribution in insets). (a) and (b) Varying frequency ratios; (c) and (d) varying amplitude ratios for $a_{0}=2400$. Note the similarity to corresponding Figs. 10 and 12. Negative spread is plotted for convenience in $(d)$. 
products may still be collimated perpendicular to the electric field polarization, but the cascade products no longer have velocities strictly in a plane.

The pairs continue to move (in an average sense) symmetrically in the frame where $\mathbf{S}=0$ due to the attractor-dragging mechanisms discussed before, but they experience strong accelerations in the direction of the electric field and thus lose their collimation perpendicular to the electric field. Their transverse momentum also grows to large values $\sim a_{0}$, causing them to spread out. Subsequently, they give rise to cascades of their own, which are fully described by our statistics (albeit with a correction factor to account for the nonzero trace of the energy-momentum tensor as the Schwinger field is approached).

A point of difference between our calculated rapidity for the frame of zero power flow (ZPF), and the one in Ref. [7] for the frame where $\mathbf{S}=0$, is that they differ by a factor of 2. This is likely because the first row of the energy-momentum tensor, the four-momentum density, was assumed to transform as a four-vector in the latter. This yields an expression for the zero-Poynting-vector frame, $\left\langle v_{x}^{(1)}\right\rangle=$ $\left(\omega_{1}^{2} a_{1}^{2}-\omega_{2}^{2} a_{2}^{2}\right) /\left(\omega_{1}^{2} a_{1}^{2}+\omega_{2}^{2} a_{2}^{2}\right)$. While the Poynting vector does not vanish in a frame traveling at $\left\langle v_{x}^{(1)}\right\rangle$, the total integrated energy-momentum flow does, if the two pulses are of the same duration and profile in the laboratory frame; the total four-momentum is indeed a four-vector. It is reasonable to expect that the frame where the total energy and momentum flow vanishes [the so-called field rest frame (FRF)] might also provide a reasonable approximation for the frame where the final distribution is symmetric. The final distribution can only be explained as a combination of several such dynamical effects. Figure 17 shows that the numerical curves do indeed typically lie between the approximations given by the ZMFZPF and FRF estimates.

\section{CONCLUSIONS}

To summarize, we have studied the nonlinear dynamics (in the classical regime) of a fermion and an electromagnetic cascade initiated by a fermion in the superposition of two counterpropagating laser pulses with different frequency or amplitude ratios. We demonstrate by Monte Carlo and PIC simulations that by tuning the frequency ratio of two laser pulses, one can control the directions of the particle jets that come out of the electromagnetic cascade. Monte Carlo simulations employed plane laser pulses, while PIC simulations presented results with temporally Gaussian (spatially uniform) beams as well as for laser pulses with Gaussian spatiotemporal profiles including the effect of an offset between the focus positions of two laser pulses. In particular, we showed how higher frequency and/or amplitude ratios give rise to jets traveling closer to the axis. This aspect of controlling the jet direction and collimation can serve as a diagnostic tool to study the electromagnetic cascade in a laboratory with the upcoming laser facilities [13-16]. We also compare our results with the spectra of the particle generated from pure vacuum decay $[7,8]$. This is especially important since particles generated from the vacuum decay can initiate further shower- or avalanche-type cascades which dominate

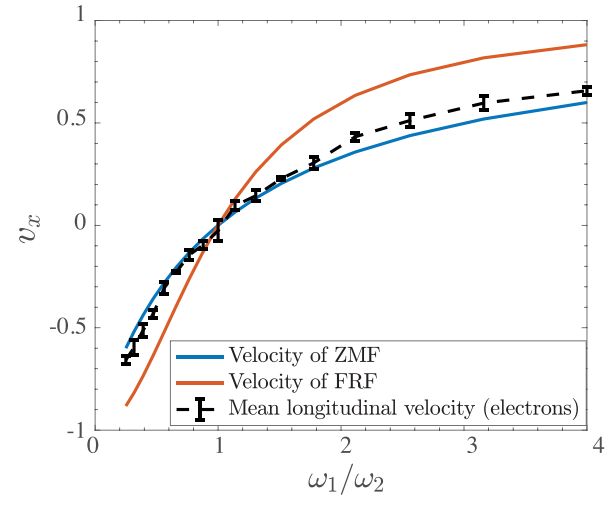

(a)

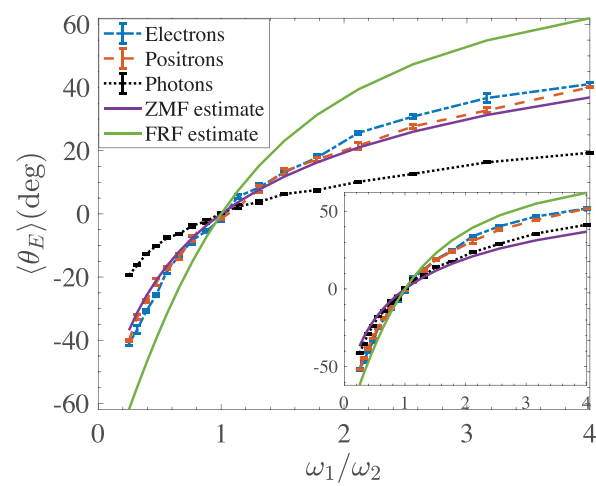

(b)

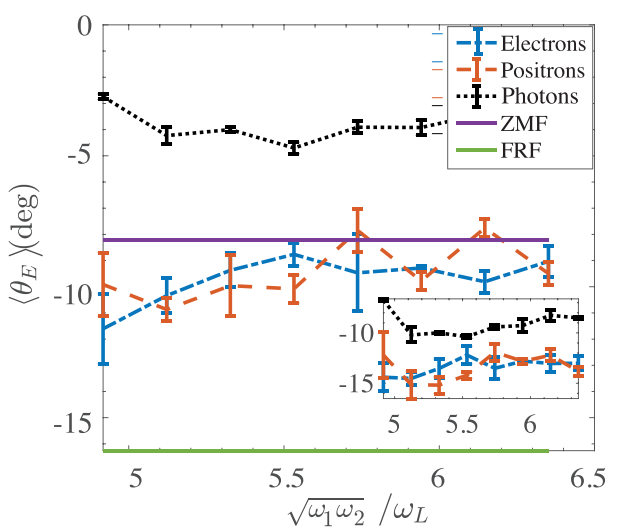

(c)

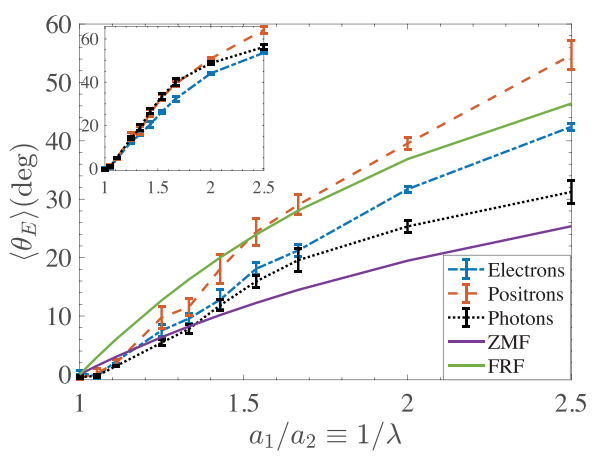

(d)

FIG. 17. (a)-(d) Several important curves, already discussed in Sec. V, lie between the ZMF-ZPF and FRF predictions. The FRF spectrum is taken from Ref. [7]. 
the jet formation in laboratory experiments. Using the criteria developed in Ref. [12], one can say that our results always indicate an avalanche-type cascade. Our results can also be exploited to distinguish between vacuum-decay products and the cascade background.

In the classical limit, we derived analytical approximations to the nonlinear dynamics, as shown in the Appendix. The classical limit is useful as long as $\chi_{e} \ll 1$ and also provides valuable insights into the particle dynamics that can help understand the simulation results in the QED regime. We explain controlling the jet properties by extending the attractor dragging to the QED limit. In the QED regime, the condition to adiabatically vary the frequencies can be relaxed, if one only considers a statistical distribution of particles, which only multiply as the cascade progresses. Fixing the laser frequencies or amplitudes at the beginning of the experiment is sufficient for achieving the desired jet properties. The numerical results often lay between the curves predicted from two considerations (zero net energy transfer vs ZPF, $\mathrm{ZMF}$, and attractor dragging), with both zero power transfer and in the case of equal amplitudes, attractor dragging, mandating symmetric motion in the ZMF. Jet properties were found to be only weakly dependent upon the individual values of frequencies or amplitudes. What is remarkable about the results obtained is that particles appear to evolve into (i.e., achieve zero longitudinal velocity in) the zero-power-flow frame for all the values of frequency and amplitude ratios explored.

Our results on jets' broadening can be interesting to understand the broadening of the jets in astrophysical scenarios. Astrophysical jets largely consist of pair plasma which is crucial for the central engine, e.g., a neutron star, to keep emitting the plasma jets continuously [57]. The central engine also emits a large quantity of low-frequency magnetic dipole radiation [58]. Astrophysical jets are also often categorized based on their angular spread [57,59]. Collimation (hence the angular spread) of astrophysical jets is not fully understood and is explained as being due to either the physical processes originating at the central engine or the interaction of the jets with the interstellar medium [60]. In both scenarios, the astrophysical jet is expected to have strong dependence on the pair-plasma density, which can be as high as Goldreich-Julian density [61]. To explain the spread of the jet at the source itself, this setup of two counterpropagating linearly polarized laser pulses with varying frequencies can exploit some parallels, e.g., a standing wave is created and the associated time-varying ponderomotive force is of low frequency for frequency ratio closer to unity. It is suggested that rotation of the central engine slows down due to the ponderomotive action of the pair-plasma currents [62]. Consequently, frequency variation in the emitted radiation occurs, and it is akin to the modulation in the ponderomotive force. Since the angular spread of the jets is affected by the frequency ratio of the two laser pulses in the simulations, one can argue that the low-frequency radiation emitted by the source engine can impact the angular spread of the emitted jets.

\section{ACKNOWLEDGMENTS}

Simulations were carried out on a virtual machine with a student subscription of Microsoft Azure platform. One of us (N.K.) acknowledges a discussion with S. Tang.

\section{APPENDIX: CLASSICAL ANALYSIS OF MOTION IN THE ANTINODAL PLANE}

Motion in the nodal and antinodal planes has been studied in some depth in the past for circularly polarized lasers [28,29]. This section presents an analysis of particle motion in the antinodal plane of a linearly polarized electromagnetic standing wave. It is easy to see that motion purely in the electric nodal plane for the setup studied in this paper and amplitude ratio unity is not interesting. The $x$ component

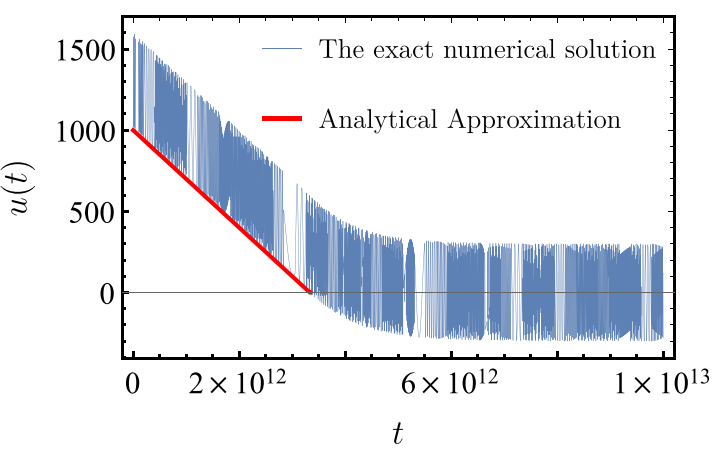

(a)

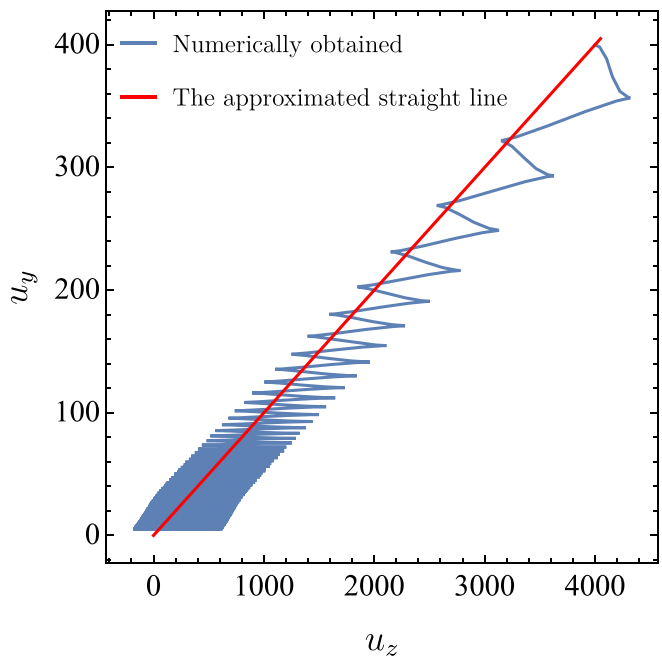

(b)

FIG. 18. (a) The momentum vs time, for a particle with $\epsilon_{\text {rad }}=$ $6.7 \times 10^{-8}, a_{0}=300$, and $u_{0}=300$ showing linear decay and rapid oscillations. (b) $2 \mathrm{D}$ motion in the electric antinodal plane, $u_{y}$ vs $u_{z}$ : Oscillations exist, but a straight line (signifying the decay) passes roughly through the origin, and the "moving center" of these oscillations, such that the ratio always oscillates about $k=0.1$. As $u_{y} \rightarrow 0$, the $z$ motion settles to the oscillatory form studied in (a). Here, $a_{0}=200, \epsilon_{\text {rad }}=6.7 \times 10^{-8}$, and $u_{y 0}=u_{z 0} / 10=400$. 
of the right-hand side of Eq. (1) at $x=(n+1 / 2) \pi, n \in \mathbb{Z}$, is $\pm \omega a_{0} v_{z} \cos (\omega t)$. Meanwhile, $d u_{x} / d t=0$. This is only consistent if $v_{z}=0$; then, motion is purely in the $y$ direction. The particle feels no force; it drifts at a constant velocity along the $y$ axis.

Consider the motion of a particle in a standing wave formed by counterpropagating laser pulses, in the electric antinodal plane $\left(x=v_{x}=0\right)$; note that it is unstable for perturbations along the direction of wave propagation. With amplitude parameter $a_{0}$ and an initial velocity along the electric field direction (say, $\hat{\mathbf{z}}$ ), the equation of motion has forcing and damping terms and reads

$$
\begin{aligned}
\frac{d \mathbf{u}}{d t}= & {\left[\frac{2}{3} \omega^{2} \gamma a_{0} \cos (\omega t)+\frac{2}{3} \omega^{2} a_{0}^{2} v_{z} \sin ^{2}(\omega t)\right.} \\
& \left.\times \omega a_{0} \sin (\omega t)\right] \hat{\mathbf{z}}-\frac{2}{3} \gamma^{2} \omega^{2} a_{0}^{2} \mathbf{v} \sin ^{2}(\omega t)\left(1-v_{z}^{2}\right) \\
& \frac{d u_{z}}{d t}=\left[\omega a_{0} \sin (\omega t)+\frac{2}{3} \omega^{2} \gamma a_{0} \cos (\omega t)\right]
\end{aligned}
$$

Any initial velocity is damped by radiation reaction, until it gets sufficiently small, and the velocity oscillates roughly in sync with the forcing term, with a phase difference. If the oscillation frequency of the forcing term $(\omega)$ is considerably larger than the characteristic damping time, or alternatively, if the magnitude of the forcing term is considerably smaller than that of the damping term, the fast oscillations can be averaged out or ignored to show how velocities decay. On approximating $\sqrt{1+u_{z}^{2}} \approx u_{z}$, and writing $u_{z}(t)=\operatorname{slow}(t)+$ envelope $(t) P(\omega t)$, where slow and envelope are slowly varying functions of time, while $P(\omega t)$ is a periodic function with frequency $\omega$, one may split $P(\omega t)$ into a Fourier series. Averaging the forcing term in Eq. (A2), we get zero. Averaging over the second term with the mentioned substitutions, only the product with the $\cos \omega t$ term in the Fourier series of $P(\omega t)$ survives. From inspection, it is clear that this term is in fact $-a_{0} \cos \omega t$. Thus we end up with the differential equation $d u_{z} / d t=-\omega^{2} a_{0}^{2} / 3$, which has a straight-line solu- tion. Note that, in this case, replacing the radiation friction by $-2 \gamma^{2} \omega^{2} a_{0}^{2} \mathbf{v} \sin ^{2}(\omega t)\left(1-v_{z}^{2}\right) / 3$, assumed to be dominant, can be a wrong approximation, even if it yields a linear decay. This term actually cancels itself with $2 \omega^{2} a_{0}^{2} v_{z} \sin ^{2}(\omega t) / 3$.

For general planar motion, the initial motion is in a straight line, until the $y$ component damps out, and particles perform forced oscillatory motion along the direction of the electric field. Again, the decay due to radiation friction can easily be analytically approximated; see Fig. 18(a). The highamplitude oscillations over the linear decay are due to the second term, which dominates when $u_{z} \gg 1$. A solution of the form $\sinh \left[\sinh ^{-1} u_{z 0}+2 \omega a_{0} \sin (\omega t) / 3\right]$ results. The decay time is $t_{\text {decay }} \sim u_{0} /\left(\omega^{2} a_{0}^{2}\right)$. Thus we need $t_{\text {decay }} \gg 1 / \omega$, i.e., $Q_{\text {freq }} \equiv u_{0} /\left(\omega a_{0}^{2}\right) \gg 1$, for the approximation to hold true. In Fig. 18(a), we have $Q_{\text {freq }} \sim 10^{5}$. We can, in fact, give up the $u_{y 0}=0$ assumption. In $2 \mathrm{D}$, after performing the average over fast oscillations and using the common approximation (valid in 1D) that the last term in the radiation reaction force dominates over all others, since $\gamma \gg 1$,

$$
\begin{aligned}
& u_{z}^{\prime}(t)=-\frac{1}{3} \gamma a_{0}^{2} \omega^{2} u_{z}(t) \sin ^{2}(\omega t)\left(1-v_{z}^{2}\right), \\
& u_{y}^{\prime}(t)=-\frac{1}{3} \gamma a_{0}^{2} \omega^{2} u_{y}(t) \sin ^{2}(\omega t)\left(1-v_{z}^{2}\right) .
\end{aligned}
$$

Taking their ratio yields $u_{z}=k u_{y}$, for some constant $k$. Substituting the relationship into Eq. (1), with the approximation that the initial velocity components, $u_{y 0}, u_{z 0} \gg 1$, we find that

$$
t=\frac{1}{1 / u_{z 0}+\omega^{2} a_{0}^{2} k^{2} t /\left(3 \sqrt{1+k^{2}}\right)} .
$$

To judge the accuracy of the approximation, we may impose the condition that the decay time must be much greater than the time period of oscillations. We get, for particles with $k \sqrt{k^{2}+1} u_{z 0}, \sqrt{k^{2}+1} u_{z 0} \gg 1, Q_{\text {freq }} \equiv$ $\sqrt{1+k^{2}} /\left(\omega a_{0}^{2} u_{z 0} k^{2}\right) \gg 1$. Figure 18 (b) plots $u_{y}$ vs $u_{z}$ for some demonstrative set of initial conditions. Particle velocities start at the top right of the plot, and the decay does occur along the straight line, with rapid oscillations which average out. Eventually, since the force along the magnetic field direction ( $y$ axis) is purely dissipative, $u_{y} \rightarrow 0, u_{z}$ then reduces to the form discussed earlier.
[1] F. A. Aharonian, Very High Energy Cosmic Gamma Radiation: A Crucial Window on the Extreme Universe (World Scientific, Singapore, 2004).

[2] F. Ehlotzky, K. Krajewska, and J. Z. Kamiński, Fundamental processes of quantum electrodynamics in laser fields of relativistic power, Rep. Prog. Phys. 72, 046401 (2009).

[3] W. T. Hill and L. Roso, Probing the quantum vacuum with petawatt lasers, J. Phys.: Conf. Ser. 869, 012015 (2017).

[4] I. V. Sokolov, N. M. Naumova, J. A. Nees, and G. A. Mourou, Pair Creation in QED-Strong Pulsed Laser Fields Interacting with Electron Beams, Phys. Rev. Lett. 105, 195005 (2010).

[5] E. N. Nerush, I. Yu. Kostyukov, A. M. Fedotov, N. B. Narozhny, N. V. Elkina, and H. Ruhl, Laser Field Absorption in SelfGenerated Electron-Positron Pair Plasma, Phys. Rev. Lett. 106, 035001 (2011).

[6] T. Grismayer, M. Vranic, J. L. Martins, R. A. Fonseca, and L. O. Silva, Laser absorption via quantum electrodynamics cascades in counter propagating laser pulses, Phys. Plasmas 23,
056706 (2016); Seeded QED cascades in counterpropagating laser pulses, Phys. Rev. E 95, 023210 (2017).

[7] L. Labun and J. Rafelski, Spectra of particles from laserinduced vacuum decay, Phys. Rev. D 84, 033003 (2011).

[8] L. Labun and J. Rafelski, Vacuum-decay time in strong external fields, Phys. Rev. D 79, 057901 (2009).

[9] A. Hartin, A. Ringwald, and N. Tapia, Measuring the boiling point of the vacuum of quantum electrodynamics, Phys. Rev. D 99, 036008 (2019)

[10] E. S. Efimenko, A. V. Bashinov, A. A. Gonoskov, S. I. Bastrakov, A. A. Muraviev, I. B. Meyerov, A. V. Kim, and A. M. Sergeev, Laser-driven plasma pinching in $e^{-} e^{+}$cascade, Phys. Rev. E 99, 031201(R) (2019).

[11] T. G. Blackburn, A. Ilderton, M. Marklund, and C. P. Ridgers, Reaching supercritical field strengths with intense lasers, New J. Phys. 21, 053040 (2019).

[12] A. Mironov, A. Fedotov, and N. Narozhny, Observable features of QED cascades in collisions of $\mathrm{GeV}$ electrons 
with intense laser pulses, J. Phys.: Conf. Ser. 826, 012029 (2017).

[13] Centre interdisciplinaire lumière extrême, http://cilexsaclay.fr.

[14] Extreme Light Infrastructure, http://www.eli-beams.eu.

[15] Vulcan, https://www.clf.stfc.ac.uk/Pages/Vulcan.aspx.

[16] XCELS, https://xcels.iapras.ru.

[17] C. Bula, K. T. McDonald, E. J. Prebys, C. Bamber, S. Boege, T. Kotseroglou, A. C. Melissinos, D. D. Meyerhofer, W. Ragg, D. L. Burke, R. C. Field, G. Horton-Smith, A. C. Odian, J. E. Spencer, D. Walz, S. C. Berridge, W. M. Bugg, K. Shmakov, and A. W. Weidemann, Observation of Nonlinear Effects in Compton Scattering, Phys. Rev. Lett. 76, 3116 (1996).

[18] D. L. Burke, R. C. Field, G. Horton-Smith, J. E. Spencer, D. Walz, S. C. Berridge, W. M. Bugg, K. Shmakov, A. W. Weidemann, C. Bula, K. T. McDonald, E. J. Prebys, C. Bamber, S. J. Boege, T. Koffas, T. Kotseroglou, A. C. Melissinos, D. D. Meyerhofer, D. A. Reis, and W. Ragg, Positron Production in Multiphoton Light-by-Light Scattering, Phys. Rev. Lett. 79, 1626 (1997).

[19] G. Sarri, K. Poder, J. M. Cole, W. Schumaker, A. Di Piazza, B. Reville, T. Dzelzainis, D. Doria, L. A. Gizzi, G. Grittani, S. Kar, C. H. Keitel, K. Krushelnick, S. Kuschel, S. P. D. Mangles, Z. Najmudin, N. Shukla, L. O. Silva, D. Symes, A. G. R. Thomas, M. Vargas, J. Vieira, and M. Zepf, Generation of neutral and high-density electron-positron pair plasmas in the laboratory, Nat. Commun. 6, 6747 (2015).

[20] A. R. Bell and J. G. Kirk, Possibility of Prolific Pair Production with High-Power Lasers, Phys. Rev. Lett. 101, 200403 (2008).

[21] M. Lobet, C. Ruyer, A. Debayle, E. d'Humières, M. Grech, M. Lemoine, and L. Gremillet, Ultrafast Synchrotron-Enhanced Thermalization of Laser-Driven Colliding Pair Plasmas, Phys. Rev. Lett. 115, 215003 (2015); M. Lobet, X. Davoine, E. d'Humières, and L. Gremillet, Generation of high-energy electron-positron pairs in the collision of a laser-accelerated electron beam with a multipetawatt laser, Phys. Rev. Accel. Beams 20, 043401 (2017); C. I. Hojbota, H. T. Kim, V. B. Pathak, and C. H. Nam, Influence of polarization on backreflected $e^{-} e^{+}$pair jets from laser-electron collision, Plasma Phys. Control. Fusion 62, 024003 (2019).

[22] U. Sinha and N. Kumar, Pair-beam propagation in a magnetized plasma for modeling the polarized radiation emission from gamma-ray bursts in laboratory astrophysics experiments, Phys. Rev. E 101, 063204 (2020); U. Sinha, C. H. Keitel, and N. Kumar, Polarized Light from the Transportation of a MatterAntimatter Beam in a Plasma, Phys. Rev. Lett. 122, 204801 (2019).

[23] J. G. Kirk, A. R. Bell, and I. Arka, Pair production in counterpropagating laser beams, Plasma Phys. Controlled Fusion 51, 085008 (2009).

[24] V. Yakimenko, S. Meuren, F. Del Gaudio, C. Baumann, A. Fedotov, F. Fiuza, T. Grismayer, M. J. Hogan, A. Pukhov, L. O. Silva, and G. White, Prospect of Studying Nonperturbative QED with Beam-Beam Collisions, Phys. Rev. Lett. 122, 190404 (2019).

[25] K. Qu, S. Meuren, and N. J. Fisch, Signature of Collective Plasma Effects in Beam-Driven QED Cascades, Phys. Rev. Lett. 127, 095001 (2021).

[26] V. I. Ritus, Quantum effects of the interaction of elementary particles with an intense electromagnetic field, J. Sov. Laser Res. 6, 497 (1985).
[27] K. Krajewska and J. Z. Kamiński, Breit-Wheeler process in intense short laser pulses, Phys. Rev. A 86, 052104 (2012).

[28] J. G. Kirk, Radiative trapping in intense laser beams, Plasma Phys. Controlled Fusion 58, 085005 (2016).

[29] T. Zh. Esirkepov, S. S. Bulanov, J. K. Koga, M. Kando, K. Kondo, N. N. Rosanov, G. Korn, and S. V. Bulanov, Attractors and chaos of electron dynamics in electromagnetic standing waves, Phys. Lett. A 379, 2044 (2015); S. V. Bulanov, T. Zh. Esirkepov, J. K. Koga, S. S. Bulanov, Z. Gong, X. Q. Yan, and M. Kando, Charged particle dynamics in multiple colliding electromagnetic waves. Survey of random walk, Lévy flights, limit circles, attractors and structurally determinate patterns, J. Plasma Phys. 83, 905830202 (2017).

[30] T. Nakamura, J. K. Koga, T. Zh. Esirkepov, M. Kando, G. Korn, and S. V. Bulanov, High-Power $\gamma$-Ray Flash Generation in Ultraintense Laser-Plasma Interactions, Phys. Rev. Lett. 108, 195001 (2012).

[31] G. Lehmann and K. H. Spatschek, Phase-space contraction and attractors for ultrarelativistic electrons, Phys. Rev. E 85, 056412 (2012).

[32] A. Gonoskov, A. Bashinov, I. Gonoskov, C. Harvey, A. Ilderton, A. Kim, M. Marklund, G. Mourou, and A. Sergeev, Anomalous Radiative Trapping in Laser Fields of Extreme Intensity, Phys. Rev. Lett. 113, 014801 (2014).

[33] M. J. A. Jansen and C. Müller, Strongly enhanced pair production in combined high- and low-frequency laser fields, Phys. Rev. A 88, 052125 (2013).

[34] A. Otto, T. Nousch, D. Seipt, B. Kämpfer, D. Blaschke, A. D. Panferov, S. A. Smolyansky, and A. I. Titov, Pair production by Schwinger and Breit-Wheeler processes in bi-frequent fields, J. Plasma Phys. 82, 655820301 (2016).

[35] J. Braß, R. Milbradt, S. Villalba-Chávez, G. G. Paulus, and C. Müller, Two-color phase-of-the-phase spectroscopy applied to nonperturbative electron-positron pair production in strong oscillating electric fields, Phys. Rev. A 101, 043401 (2020).

[36] A. I. Titov, H. Takabe, and B. Kämpfer, Nonlinear BreitWheeler process in short laser double pulses, Phys. Rev. D 98, 036022 (2018).

[37] S. Augustin and C. Müller, Nonperturbative Bethe-Heitler pair creation in combined high- and low-frequency laser fields, Phys. Lett. B 737, 114 (2014).

[38] S. C. Wilks, J. M. Dawson, and W. B. Mori, Frequency Up-Conversion of Electromagnetic Radiation with Use of an Overdense Plasma, Phys. Rev. Lett. 61, 337 (1988).

[39] V. Leidel, Laser scattering calculations in pair producing plasmas, Bachelor thesis, Ruprecht-Karls-Universität, Heidelberg, 2019.

[40] K. Qu and N. J. Fisch, Laser frequency upconversion in plasmas with finite ionization rates, Phys. Plasmas 26, 083105 (2019).

[41] M. Altarelli, The European x-ray free-electron laser facility in Hamburg, Nucl. Instrum. Methods Phys. Res., Sect. B 269, 2845 (2011).

[42] B. W. J. McNeil and N. R. Thompson, X-ray free-electron lasers, Nat. Photonics 4, 814 (2010).

[43] L. D. Landau and E. M. Lifshitz, The Classical Theory of Fields (Addison-Wesley, Reading, MA, 1971), Vol. 2.

[44] P. Gibbon, Short Pulse Laser Interactions with Matter: An Introduction (World Scientific, Singapore, 2005). 
[45] A. I. Akhiezer and R. V. Polovin, Theory of wave motion of an electron plasma, Sov. Phys. JETP 3, 696 (1956).

[46] Z. Gong, R. Hu, Y. Shou, B. Qiao, C. Chen, F. Xu, X. He, and $\mathrm{X}$. Yan, Radiation reaction induced spiral attractors in ultraintense colliding laser beams, Matter Radiat. Extremes 1, 308 (2016).

[47] A note of caution: The difference between attracting sets and attractors is often a matter of pedagogy. While often an attractor is an attracting set which follows an additional set of constraints, it is common not to have the precise set of constraints, or even not to impose such constraints at all. In our treatment here, we shall use the terminology interchangeably.

[48] J. Milnor, On the concept of attractor, Commun. Math. Phys. 99, 177 (1985).

[49] It is not clear whether Eq. (2) actually holds true for a given (thermal or cold) distribution of several particles. It might seem intuitive to assume that it may hold true based on the reasoning we have presented (attractor dragging and zero net power flow in the ZMF). However, notice the situation of varying frequency ratios in the ZMF: Particles are hurled with different velocities in a standing electromagnetic wave formed by counterpropagating pulses with equal frequencies and amplitudes. What we shall see will pertain to the fact that the particles, regardless of their initial velocity, come to rest on average in this frame. This needs substantiation, for it is not clear whether a directionally biased initial condition in the ZMF should lead to an unbiased, "thermal" distribution in the ZMF. In principle, it can be possible for particles to preferentially move, on average, in the direction of their initial velocity; the orbits of the attracting sets can also support this motion.

[50] R. Duclous, J. G. Kirk, and A. R. Bell, Monte Carlo calculations of pair production in high-intensity laser-plasma interactions, Plasma Phys. Controlled Fusion 53, 015009 (2010).
[51] S. Tiwary, 3d Monte Carlo: MATLAB simulation code, https:// github.com/siddharth110200/3d-Monte-Carlo, 2020.

[52] The Monte Carlo Algorithm for Photon Emission, and Radiation Tables, https://smileipic.github.io/Smilei/radiation_loss. html.

[53] The Monte Carlo Algorithm for Pair Production, and Related Tables, https://smileipic.github.io/Smilei/multiphoton Breit_Wheeler.html.

[54] U. Levy and Y. Silberberg, Weakly diverging to tightly focused Gaussian beams: a single set of analytic expressions, J. Opt. Soc. Am. A 33, 1999 (2016).

[55] J. Derouillat, A. Beck, F. Pérez, T. Vinci, M. Chiaramello, A. Grassi, M. Flé, G. Bouchard, I. Plotnikov, N. Aunai, J. Dargent, C. Riconda, and M. Grech, SMILEI: A collaborative, open-source, multi-purpose particle-in-cell code for plasma simulation, Comput. Phys. Commun. 222, 351 (2018).

[56] C. W. Misner, K. S. Thorne, and J. A. Wheeler, Gravitation (Freeman, San Francisco, 1973).

[57] W. Kundt, Bipolar flows, in Astrophysics: A New Approach (Springer, Berlin, 2005), pp. 119-126.

[58] J. E. Gunn and J. P. Ostriker, Magnetic dipole radiation from pulsars, Nature (London) 221, 454 (1969).

[59] H. C. Spruit, Theory of magnetically powered jets, in The Jet Paradigm: From Microquasars to Quasars, edited by T. Belloni (Springer, Berlin, 2010), pp. 233-263.

[60] E. G. Blackman and S. V. Lebedev, Persistent mysteries of jet engines, formation, propagation, and particle acceleration: have they been addressed experimentally?, arXiv:2009.08057 [astro-ph.HE].

[61] P. Goldreich and W. H. Julian, Pulsar electrodynamics, Astrophys. J. 157, 869 (1969).

[62] V. S. Beskin, A. V. Gurevich, Ya. N. Istomin, and J. Arons, Physics of the Pulsar Magnetosphere (Cambridge University Press, Cambridge, 1994). 